\title{
Three Dimensional Dynamics of a Flexible Motorised Momentum Exchange Tether
}

\author{
N.A Ismail ${ }^{1 \dagger}$ and M.P Cartmell ${ }^{2}$ \\ ${ }^{1}$ School of Aerospace Engineering, Universiti Sains Malaysia, 14300 Nibong Tebal, \\ Pulau Pinang Malaysia. \\ ${ }^{2}$ Department of Mechanical Engineering, University of Sheffield, S1 3JD, Sheffield, \\ United Kingdom. \\ E-mail: aenorilmi@usm.my,m.cartmell@sheffield.ac.uk
}

\begin{abstract}
This paper presents a new flexural model for the three dimensional dynamics of the Motorised Momentum Exchange Tether (MMET) concept. This study has uncovered the relationships between planar and nonplanar motions, and the effect of the coupling between these two parameters on pragmatic circular and elliptical orbits. The tether sub-spans are modelled as stiffened strings governed by partial differential equations of motion, with specific boundary conditions. The tether sub-spans are flexible and elastic, thereby allowing three dimensional displacements. The boundary conditions lead to a specific frequency equation and the eigenvalues from this provide the natural frequencies of the orbiting flexible motorised tether when static, accelerating in monotonic spin, and at terminal angular velocity. A rotation transformation matrix has been utilised to get the position vectors of the system's components in an assumed inertial frame. Spatio-temporal coordinates are transformed to modal coordinates before applying Lagrange's equations, and preselected linear modes are included to generate the equations of motion. The equations of motion contain inertial nonlinearities which are essentially of cubic order, and these show the potential for intricate intermodal coupling effects. A simulation of planar and non-planar motions has been undertaken and the differences in the modal responses, for both motions, and between the rigid body and flexible models are highlighted and discussed.
\end{abstract}

\footnotetext{
${ }^{\dagger}$ Corresponding author. Tel.: +604-5995944; fax: +604-5996911
} 


\section{Introduction}

The Motorised Momentum Exchange Tether or MMET was first proposed by Cartmell in 1996 and a summary of the model was published in 1998 [1]. The MMET is a symmetrical system with motorised spin-up operating against a counter inertia. The inclusion of a motor, assumed to be powered by electricity from a solar panel or a fuel cell, provides an opportunity for generating additional velocity change.

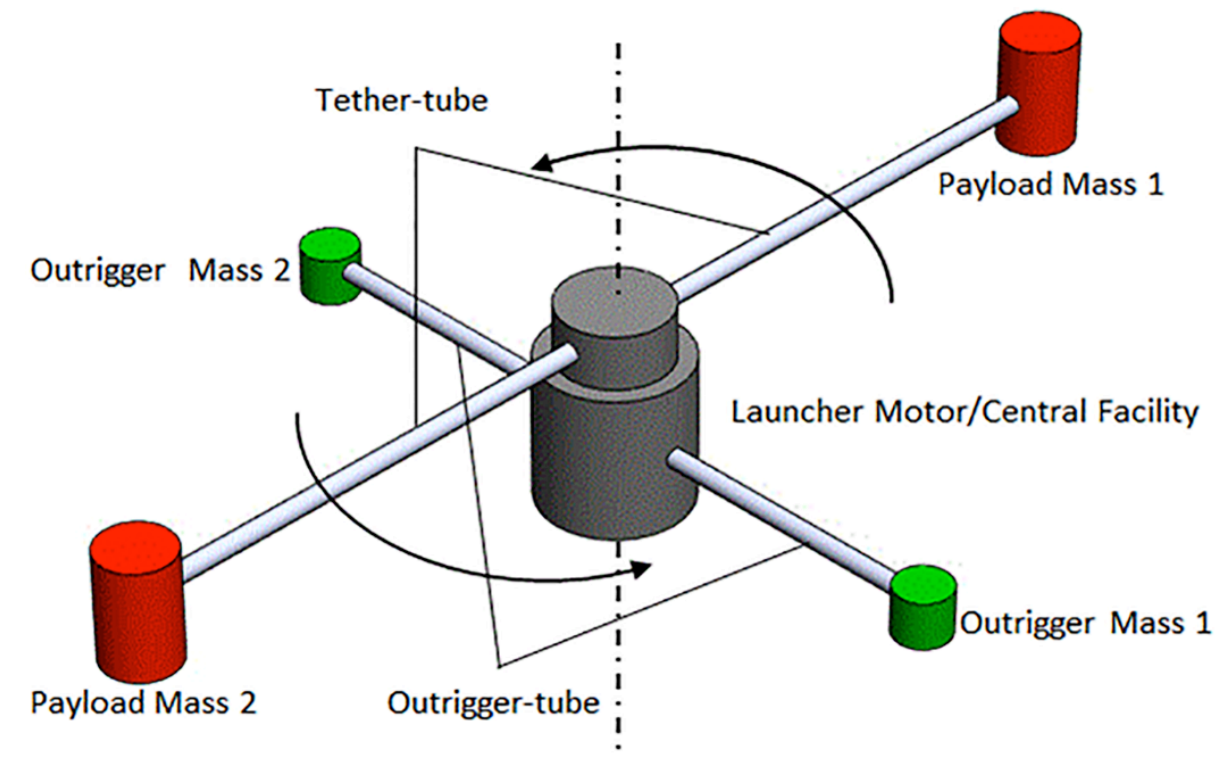

Figure 1: Symmetrical Motorised Momentum Exchange Tether

A tether should be modelled to the level of accuracy required for the specific objectives to be achieved, so that the necessary analysis can then be developed. A simple model reduces the complexity of the problem, but potentially introduces a lack of accuracy since important phenomena may not be taken into account. The simplest model describing rigid body motion is based on a massless rigid rod in which bending and stretching are negligible [2, 3, 4]. Previous studies by Modi et al. [5], Puig-Suari and Longuski [6], and Ziegler and Cartmell [7] have all been based on the assumption that the tether is a massive rigid rod. The benefit of including the tether's mass is to generate more accurate mission data for quantitative analysis. Fujii and Ishijima [8] enhanced the tether rigid body model into the form of an extensible, massless rod in order to include the effect of the first longitudinal stretch mode to the system. In [9], 
He et al. disregarded the flexibility and elasticity of the tether and have modelled it as uniform in mass in order to study the stability of the tether in depth. The stability study was used as an input for range rate control for tether deployment and retrieval. The dumbbell tether model has also been used in the recent study of tether control by Iñarrea et al. [10] on the stabilisation of an electrodynamics tether in an elliptic inclined orbit. A three mass-tethered satellite model consisting of two end bodies and a climber was used in [11] and [12]. In the study of a multi-tethered satellite formation system Cai et al. [13] used a massless tether connected together with point masses, and Razzaghi et al. [14] modelled the system as three masses connected by a straight, uniform and inelastic tether with the inclusion of the $\mathrm{J} 2$ perturbation and aerodynamic drag.

The next category is represented by a sequence of elements which allows some form of flexibility in the model where $[15,16,17]$ studied a lumped mass model connected by massless springs. A bead model was used by Avanzini and Fedi [18] for massive a tether in modelling a multi-tethered satellite formation. A one-dimensional discreet tether modelled by Kunugi et al. [19] included torsional and bending vibration to investigate the used of smart film sensors in a tape tether. Biswell et al. [20] used a different model to demonstrate flexible behaviour for aerobraking tethers. The tether is modelled as hinged rigid bodies which are connected with massless springs and dampers in order to be able to model precisely the aerodynamics and gravitational forces, and the moment, with a limited number of elements which, in turn, give a reduction in the computational cost. Two examples of motion, the swinging of a cable and the plane motion of a space vehicle with a deploying tether system on orbit, have been studied to verify the mathematical model and computer code, and also to estimate the accuracy of calculation [17]. Cartmell and McKenzie [21] remarked on the important point made by Danilin et al. [17] that tether element forces cannot be compressive, so the numerical solution algorithm has to accommodate this. Netzer and Kane [22] and Kumar [23] confirmed that the more elements that are used, the more closely it will represent a continuous system. In fact, Kim and Vadali [24] showed that the bead model has the advantage of capturing most of the phenomena of the problem in comparison with the more computationally expensive continuum model. 
The other category for tether modelling is the continuous massive tether. Such a model can be elastic or inextensible. This approach is in general considered to be a way to model the tether, and is found in most of the nonlinear literature [25, 26, 27]. French et al. [28] have shown that the effect of adding tether mass and elasticity in their continuum tether model did not make a significant impact on the performance of an asteroid mitigation system. The recent study of Lee et al. [29] included a reeling mechanism in their high fidelity model of two rigid bodies connected by an elastic tether. The reeling mechanism captured the coupling interaction between the tether reeling and rotational dynamics.

The modelling strategy for the MMET, to date, has also been to use rigid body modelling in order to keep the resulting analytical models as tractable as possible. This was founded on the fair and reasonable justification that centripetal stiffening eliminates some of the flexural response, and that much of the ensuing behaviour will therefore be similar to that of a rigid body. Three dimensional rigid body tether models were derived by Ziegler and Cartmell [7] and Ziegler [30] to explain successfully many of the fundamental motions possible for a motorised momentum exchange tether. Zukovic et al. [31] used essentially the same type of model to study the dynamics of a parametrically excited planar tether. However, the previous modelling strategies $[1,7,30,31]$ discounted the flexural characteristics of the tether sub-spans, and so some important phenomena could not be captured because of this. A further development, by Chen and Cartmell [32] which has been using the spring mass model for the MMET, has shown that incorporating limited flexibility, in the form of an axial stretch coordinate, uncovers significant axial oscillations, with obvious relevance to payload release and capture scenarios. Ismail and Cartmell [33] studied a continuous two dimensional flexible model of the MMET, and this current study presents a three dimensional model of a flexible MMET in order to investigate the dynamics of a tether that may not otherwise be captured by a rigid body model.

\section{System overview}

Figure 2 shows the motions of a three dimensional flexible model of an MMET on orbit. The centre of the Earth is defined by the origin of the $X-Y-Z$ coordinate system and the tether's centre of mass is at the origin of the relative 
rotating co-ordinate system, $X_{1}-Y_{1}-Z_{1}$. The $X-Y-Z$ plane and the $X_{1}-Y_{1}-Z_{1}$ plane lie within the orbital plane.

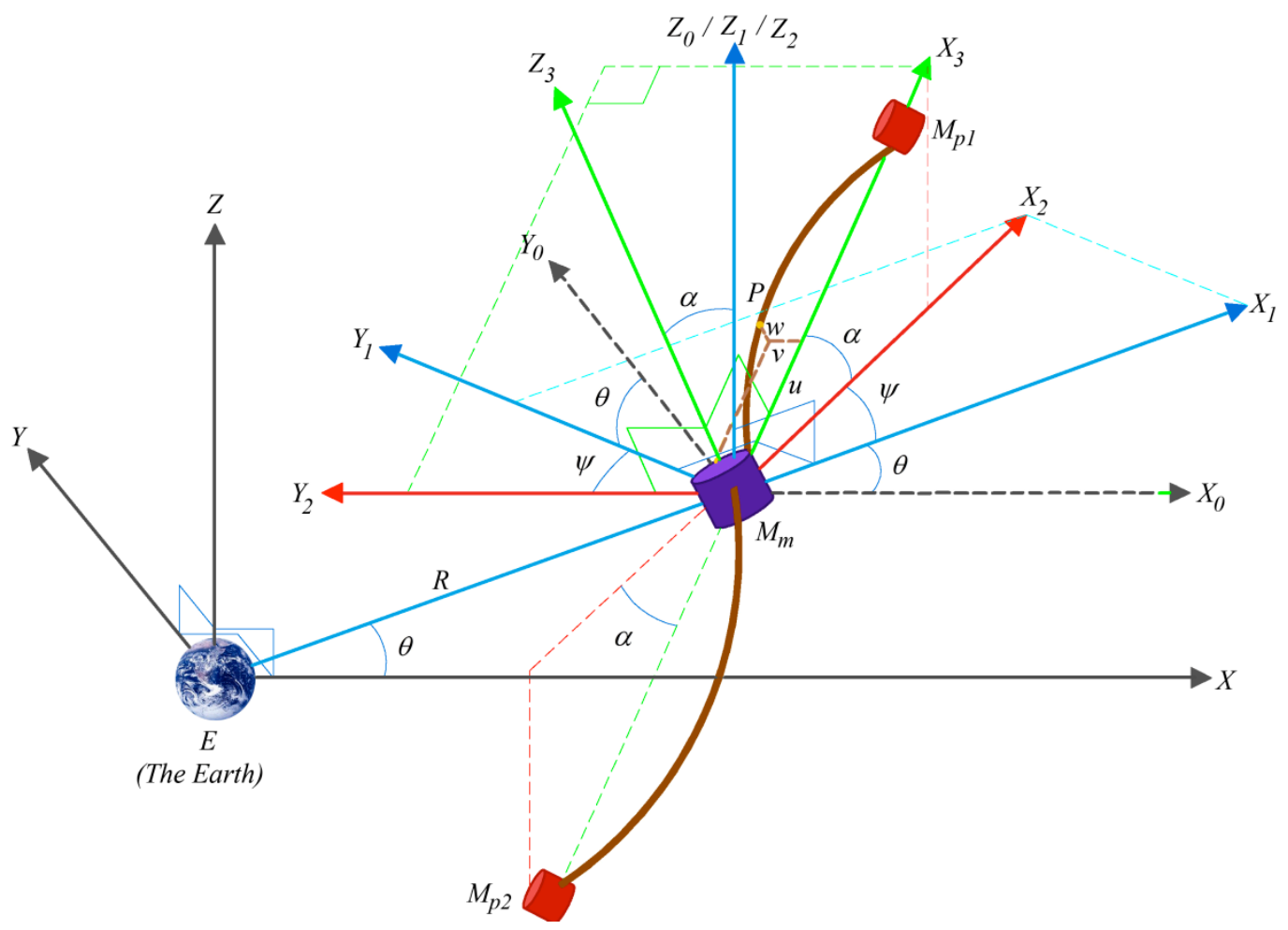

Figure 2: Three dimensional flexible schematic model of the MMET

The $X$ axis is aligned to the direction of the perigee of the orbit and the $X_{1}$ axis aligned along $R$, which is the distance from the central facility to the centre of the Earth. The angle from the direction of perigee of the orbit to the centre of mass is given by the true anomaly, $\theta$. The in-plane angle $\psi$, is the angle from the $X_{l}$ axis to the position of the tether on the orbital plane. The payload masses, $M_{P 1}$ and $M_{P 2}$ are connected by the tether sub-spans to the central facility, $M_{m}$, and the components of flexibility of the MMET are described by the displacements of the tether in the axial and transverse directions, by $u, v$, and $w$.

The elastic displacements $u, v$ and $w$ are functions dependent both on space and time and can be separated as follows, with recourse to the Bubnov-Galerkin [34] method,

$$
u(x, t)=\sum_{i=1}^{n} \phi(x) q_{1}(t), \quad v(x, t)=\sum_{i=1}^{n} \xi(x) q_{2}(t), \quad w(x, t)=\sum_{i=1}^{n} \beta(x) q_{3}(t)
$$


where the $\phi(x), \xi(x)$ and $\beta(x)$ are spatial linear mode shape functions and $q_{1}(\mathrm{t}), q_{2}(\mathrm{t})$, and $q_{3}(\mathrm{t})$, are time dependent modal coordinates. Assuming that the payload and central facility are so massive that the tether sub-spans experience them as being equivalent to built-in ends then the mode shape functions are given by,

$$
\phi(x)=\xi(x)=\beta(x)=\sin \frac{\pi x}{L}
$$

This approach for the boundary conditions is echoed in the work of Luo et al. [35], where the same assumption of fixed end boundary conditions is used to get the mode shape functions, thereby simplifying the derivation of the equations of motion for a stretched spinning tether.

The local position of a point mass $P$, in Figure 2 is transformed to inertial coordinates by rotating and translating the position vector. The position of the central facility $\mathrm{Mm}$, is translated through distance $R$, then rotated through angle $\theta$, as in Figure 2. The system is further rotated about the $Z_{0}$ axis through angle $\psi$. Finally, the system is rotated about the $Y_{2}$ axis through angle $\alpha$ to give a basis for the full nonplanar motion of the MMET. These rotations can be stated in a rotation matrix denoted by $R_{n, k}$ where $n$ refers to the axis of rotation, and $k$ is the rotation angle. Therefore the complete rotation matrix from local coordinates to the inertial coordinates is defined as,

$$
R_{Z Y}=R_{Z, \psi+\theta} \cdot R_{Y, \alpha}=\left(\begin{array}{ccc}
\cos \alpha \cos (\theta+\psi) & -\sin (\theta+\psi) & -\sin \alpha \cos (\theta+\psi) \\
\cos \alpha \sin (\theta+\psi) & \cos (\theta+\psi) & -\sin \alpha \sin (\theta+\psi) \\
\sin \alpha & 0 & \cos \alpha
\end{array}\right)
$$

\section{Cartesian Components}

The initial coordinates of the payloads and central facility with respect to the local origin are given by,

$$
\left\{\begin{array}{l}
x_{P 1} \\
y_{P 1} \\
z_{P 1}
\end{array}\right\}=\left\{\begin{array}{c}
R \cos \theta+L \cos (\psi+\theta) \\
R \sin \theta+L \sin (\psi+\theta) \\
L \sin \alpha
\end{array}\right\}
$$




$$
\begin{gathered}
\left\{\begin{array}{l}
x_{P 2} \\
y_{P 2} \\
z_{P 3}
\end{array}\right\}=\left\{\begin{array}{c}
R \cos \theta-L \cos (\psi+\theta) \\
R \sin \theta-L \sin (\psi+\theta) \\
-L \sin \alpha
\end{array}\right\} \\
\left\{\begin{array}{l}
x_{m m} \\
y_{m m} \\
z_{m m}
\end{array}\right\}=\left\{\begin{array}{c}
R \cos \theta \\
R \sin \theta \\
0
\end{array}\right\}
\end{gathered}
$$

Applying equation (3) to the position of an arbitrary point $P$ along the tether gives the new position coordinates in terms of the $x, y, z$ components, for non-planar motion,

$$
\begin{aligned}
& \left\{\begin{array}{l}
x_{t 1} \\
y_{t 1} \\
z_{t 1}
\end{array}\right\}=\left\{\begin{array}{c}
R \cos \theta+(u+x) \cos \alpha \cos (\theta+\psi)-v \sin (\theta+\psi)-w \sin \alpha \cos (\theta+\psi) \\
R \sin \theta+(u+x) \cos \alpha \sin (\theta+\psi)+v \cos (\theta+\psi)-w \sin \alpha \cos (\theta+\psi) \\
w \cos \alpha+(u+x) \sin \alpha
\end{array}\right\} \\
& \left\{\begin{array}{l}
x_{t 2} \\
y_{t 2} \\
z 2
\end{array}\right\}=\left\{\begin{array}{c}
R \cos \theta-(u+x) \cos \alpha \cos (\theta+\psi)+v \sin (\theta+\psi)+w \sin \alpha \cos (\theta+\psi) \\
R \sin \theta-(u+x) \cos \alpha \sin (\theta+\psi)-v \cos (\theta+\psi)+w \sin \alpha \cos (\theta+\psi) \\
-w \cos \alpha-(u+x) \sin \alpha
\end{array}\right\}
\end{aligned}
$$

It should be noted that the arguments denoting the dependency of $u, v, w$ on $x$ and $t$ have been dropped in the equations above, purely for notational clarity and simplicity.

\section{Energy Expressions}

The Kinetic energy for translational motion of the three dimensional system can be stated as follows,

$$
\begin{aligned}
& T_{\text {trans }}=\frac{1}{2} M_{P 1}\left(\dot{x}_{P 1}^{2}+\dot{y}_{P 1}^{2}+\dot{z}_{P 1}^{2}\right)+\frac{1}{2} M_{P 2}\left(\dot{x}_{P 2}^{2}+\dot{y}_{P 2}^{2}+\dot{z}_{P 2}^{2}\right)+\frac{1}{2} M_{m}\left(\dot{x}_{m m}^{2}+\dot{y}_{m m}^{2}+\dot{z}_{m m}^{2}\right) \\
& +\int_{0}^{L} \frac{1}{2} \rho A\left(\dot{x}_{t 1}^{2}+\dot{y}_{t 1}^{2}+\dot{z}_{t 1}^{2}\right) d x+\int_{0}^{L} \frac{1}{2} \rho A\left(\dot{x}_{t 2}^{2}+\dot{y}_{t 2}^{2}+\dot{z}_{t 2}^{2}\right) d x
\end{aligned}
$$

and the rotational kinetic energy is for the system is,

$$
\begin{aligned}
& T_{r o t}=\frac{1}{2}\left(I_{X_{P 1}}+I_{X_{P 2}}+I_{X_{m m}}+I_{X_{t 1}}+I_{X_{t 2}}\right)\left(\dot{\gamma}^{2}\right)+\frac{1}{2}\left(I_{Y_{P 1}}+I_{Y_{P 2}}+I_{Y_{m m}}+I_{Y_{t 1}}\right. \\
& \left.+I_{Y_{t 2}}\right)\left(\dot{\alpha}^{2}\right)+\frac{1}{2}\left(I_{Z_{P 1}}+I_{Z_{P 2}}+I_{Z_{m m}}+I_{Z_{t 1}}+I_{Z_{t 2}}\right)(\dot{\psi}+\dot{\theta})^{2}
\end{aligned}
$$


The total kinetic energy for this flexible model of the tether is given by the summation of equation (9) and equation (10). Ziegler and Cartmell [7] considered the principal potential energy for the system to consist of gravitational energy. In this flexible model, the tether has additional potential energy due to elasticity derived from considering the strains which are introduced.

Therefore, the total potential energy can be stated as follows,

$$
\begin{aligned}
& U_{G}=-\frac{\mu M_{P 1}}{\sqrt{R^{2}+L^{2}+2 L R \cos \alpha \cos \varphi}}-\frac{\mu M_{P 2}}{\sqrt{R^{2}+L^{2}-2 L R \cos \alpha \cos \varphi}}-\frac{\mu M_{m}}{R} \\
& -\sum_{i=1}^{N} \frac{\mu \rho A R L}{N \sqrt{R^{2}+\left[\frac{(2 i-1) L}{2 N}\right]^{2}+\frac{(2 i-1) R L}{2 N} \cos \alpha \cos \psi}} \\
& -\sum_{i=1}^{N} \frac{\mu \rho A R L}{N \sqrt{R^{2}+\left[\frac{(2 i-1) L}{2 N}\right]^{2}-\frac{(2 i-1) R L}{2 N} \cos \alpha \cos \psi}} \\
& +\int_{0}^{l}\left[\frac{1}{2} \frac{T_{o}^{2}}{E A}+T_{o}\left(u^{\prime}+\frac{1}{2}\left(v^{\prime 2}+w^{\prime 2}\right)-\frac{1}{2} u^{\prime}\left(v^{\prime 2}+w^{\prime 2}\right)+\frac{1}{2} u^{\prime 2}\left(v^{\prime 2}+w^{\prime 2}\right)-\frac{1}{8}\left(v^{\prime 2}+w^{\prime 2}\right)^{2}\right.\right. \\
& \left.\left.+\frac{5}{8} u^{\prime 4}\right)+\frac{1}{2} E A\left(u^{\prime 2}+u^{\prime}\left(v^{\prime 2}+w^{\prime 2}\right)-u^{\prime 2}\left(v^{\prime 2}+w^{\prime 2}\right)+\frac{1}{4}\left(v^{\prime 2}+w^{\prime 2}\right)^{2}\right)\right] d x
\end{aligned}
$$

where $T_{o}$ is the tension when the tether is in the nominal configuration. This comes from centripetal effects in the rotating tether. Therefore the nominal tension $T_{o}$ is given by,

$$
T_{o}=\left(M_{p} L+\int_{0}^{L} \rho A x d x\right) \psi^{2}
$$

$\mathrm{N}$ is a counter for the number of discrete tether mass elements needed to approximate the continuum model and also to overcome a numerical singularity at $\psi=\pi$. Ziegler [30] showed that in general $N=10$ to 15 is a sufficiently fine discretisation for accurate representation of the potential energy of the sub-span. 


\section{Equations of Motion}

From this point the equations of motions can be derived using Lagrange's equations in the common undamped form as follows,

$$
\frac{d}{d t}\left(\frac{\partial T}{\partial \dot{q}_{k}}\right)-\frac{\partial T}{\partial q_{k}}+\frac{\partial U}{\partial q_{k}}=\widetilde{Q}_{k}
$$

A damped motorised tether was previously studied by Gandara [36], where the damping in the system was considered to be due to imperfect bearings in the motor and transmission, and so general frictional heat dissipation was included in the derivation of the equations of motion. The presence of damping based on this reasonable assumption was not found to have much qualitative effect on the results that were obtained, but slowed the computations down very considerably. So, in this current study, given that the flexibility of the tether has already introduced a great complexity into the system, damping is excluded in order to retain some computational tractability. The model by Ziegler [30] also excluded damping in order for a comparison to be made between the flexible and rigid models. The equations of motion for three dimensional flexible model have been derived by substituting and differentiating the energy expression for use in Lagrange's Equation. There are eight generalised coordinates,

$$
\left(q_{k}\right)=\left(\psi, \alpha, \theta, \gamma, R, q_{1}, q_{2}, q_{3}\right)^{T}
$$

where the first four refer to the rotational motion and the rest define translations of the system. The generalised forces given by Ziegler [30] are used here in identical form because the three dimensionality of that model is essentially preserved,

$$
\left(\begin{array}{l}
Q_{\psi} \\
Q_{\alpha} \\
Q_{\theta} \\
Q_{R} \\
Q_{\gamma}
\end{array}\right)=\left(\begin{array}{c}
\tau \cos \gamma \cos \alpha \\
\tau \sin \gamma \\
0 \\
0 \\
0
\end{array}\right)
$$

The motor torque $\tau$ is applied to spin up the tether so that it can be forced to reach the desired angular velocity before release of the payload. Code written in the Mathematica $^{\mathrm{TM}}$ software was used for deriving and integrating the equations of 
motion, together with the application of the equation solver NDSolve to find a numerical solution to these ordinary differential equations.

\section{Tether simulation}

Four operating conditions have been considered in this study of the tether's motion on orbit. The conditions are as follows,

i. Circular orbit, unmotorised (no torque is applied to the system). Therefore only the initial conditions are driving this version of the model.

ii. Circular orbit, motorised. A torque is applied and the effect of this dominates the motion of the system.

iii. Elliptical orbit, unmotorised (no torque is applied to the system). Once again, only the initial conditions are driving this version of the model.

iv. Elliptical orbit, motorised. A torque is applied once more and then this dominates the motion of the system.

In each condition the results for simulation of the flexible tether motion when on orbit are compared with those of the rigid body model of Ziegler and Cartmell [7]. The performance of both models was compared in order to find structural differences in the response over chosen integration times.

Unless stated otherwise all the results were generated using the following established data [1], [7], [30], [33]: $L=10 \mathrm{~km}, M_{p}=1000 \mathrm{~kg}, M_{m}=5000 \mathrm{~kg}, R=6728$ $\mathrm{km}, r_{m}=r_{p}=0.5 \mathrm{~m}, E=113 \mathrm{GPa}, \mu=3.9877848 \times 1014 \mathrm{~m}^{3} / \mathrm{s}^{2}, A=62.83 \times 10-6 \mathrm{~m}^{2}$, and $\rho=970 \mathrm{~kg} \mathrm{~m}^{-3}$. The data is based on Spectra 2000 as a good candidate material choice.

\subsection{Circular Orbit}

Simulations for the tether on a circular orbit are carried out using following initial conditions, taken from Ziegler [30]:

$\psi(0)=0.9 \mathrm{rad}, \dot{\psi}(0)=0 \mathrm{rad} / \mathrm{s}, \alpha(0)=0.01 \mathrm{rad}, \dot{\alpha}(0)=0 \mathrm{rad} / \mathrm{s}, q_{1}(0)=q_{2}(0)=q_{3}(0)=0 \mathrm{~m}$ and $\dot{q}_{1}(0)=\dot{q}_{2}(0)=\dot{q}_{3}(0)=0 \mathrm{~m} / \mathrm{s}$ 
Figure 3 shows the responses of the flexible tether model in comparison with the rigid body model, both on a circular orbit.

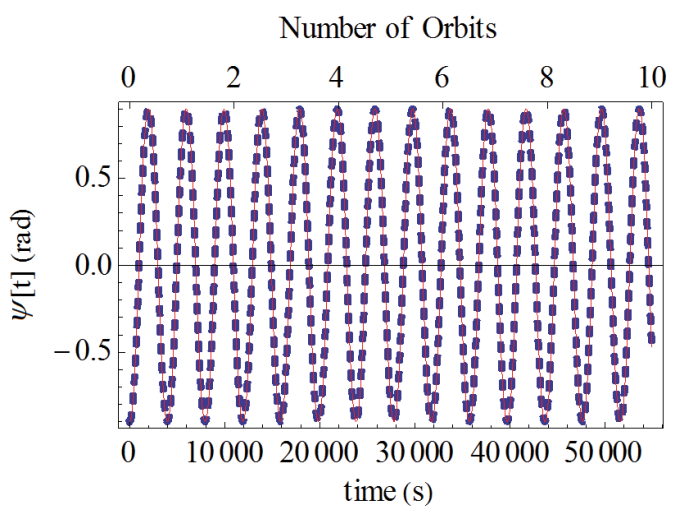

(a)

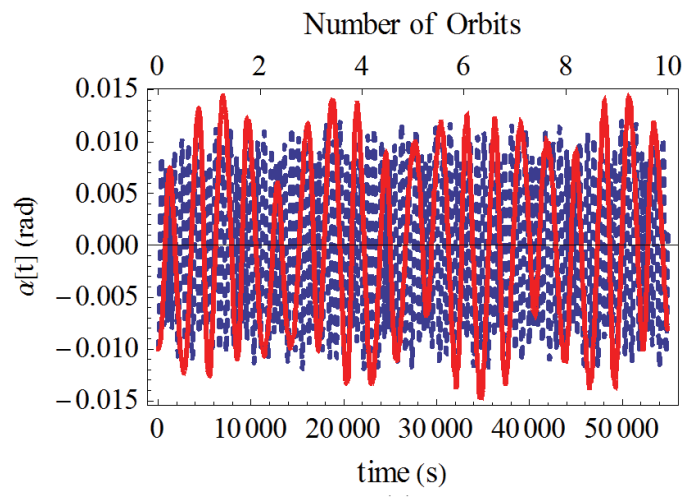

(c)

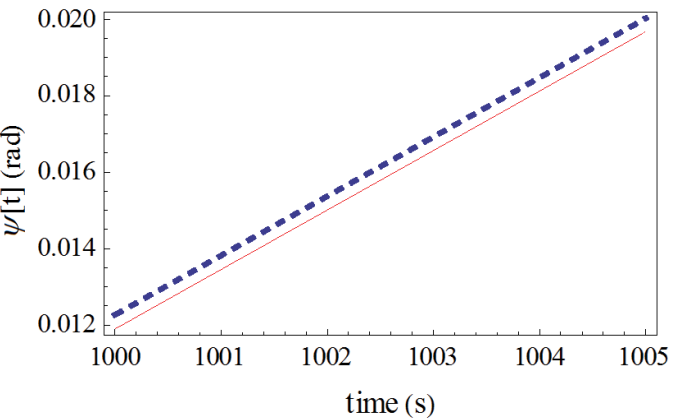

(b)

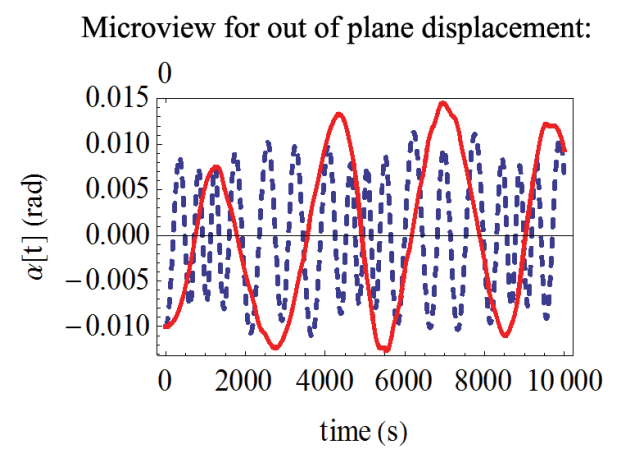

(d)

Figure 3: Responses of rigid body tether (dashed) and flexible tether (line) on a circular orbit with zero torque. (a) and (b) angular displacement and angular velocity within 10 orbits , (c) Non-planar motions in 10 orbits, and (d) microview for non-planar motion .

Both models show a very similar response for planar motion, and minor differences are only obvious within a smaller range of simulation time, as in Figure 3 (b). However, a significant difference between both models is shown for non-planar motion, in Figure 3 (c), where the flexible model oscillates at a lower frequency and reaches higher peak amplitudes as compared to those of the rigid body model.

With the application of 2.5 MNm torque, both models reach the spin-up condition, and in Figure 4 the rigid body model shows a higher rate of planar motion as compared to that of the flexible system, as shown in Figure 4 (a) and (b). As in the untorqued condition, a significant difference is evident in the non-planar motion for both models in Figure 3 (c) and (d), but not in the torqued condition in Figure 4 (c) 
and (d). Both models show decaying responses, but the rigid body model has a higher frequency and amplitude for the first eight orbits as compared to those of the flexible model shown in Figure 4 (d).

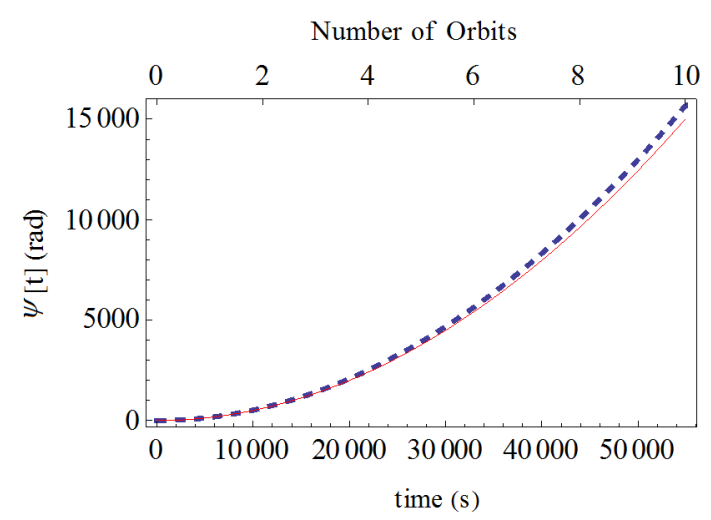

(a)

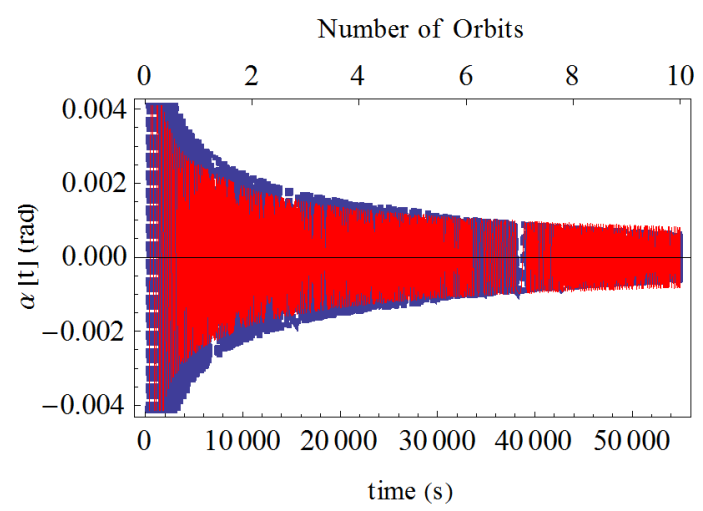

(c)

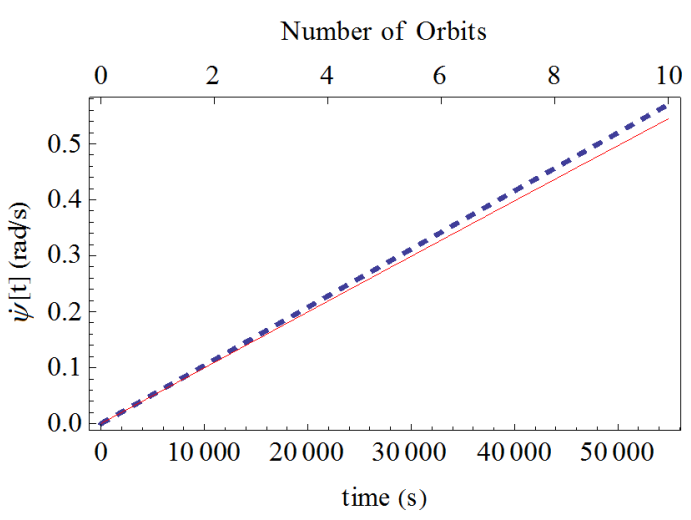

(b)

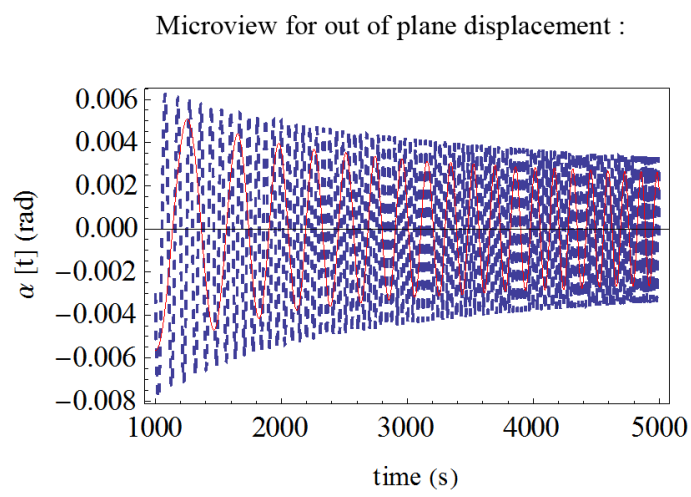

(d)

Figure 4 : Responses of rigid body tether (dashed) and flexible tether (line) with $2.5 \mathrm{MNm}$ Torque. (a) and (b) angular displacement and angular velocity within 10 orbits, (c) Non-planar motions within 10 orbits, and (d) Microview for non-planar motion.

The three dimensional displacements in the longitudinal, lateral and transverse directions are shown in Figure 5 which compares the displacement in the free vibration condition and in the torqued condition. The longitudinal, transverse and lateral displacements are oscillating with peak amplitudes of 0.008, 45 and 40 metres for the first condition. With the application of $2.5 \mathrm{MNm}$ of torque, the longitudinal displacement increases monotonically, whilst the transverse and lateral displacements experience amplitudes that are decaying over time. 
a) without torque
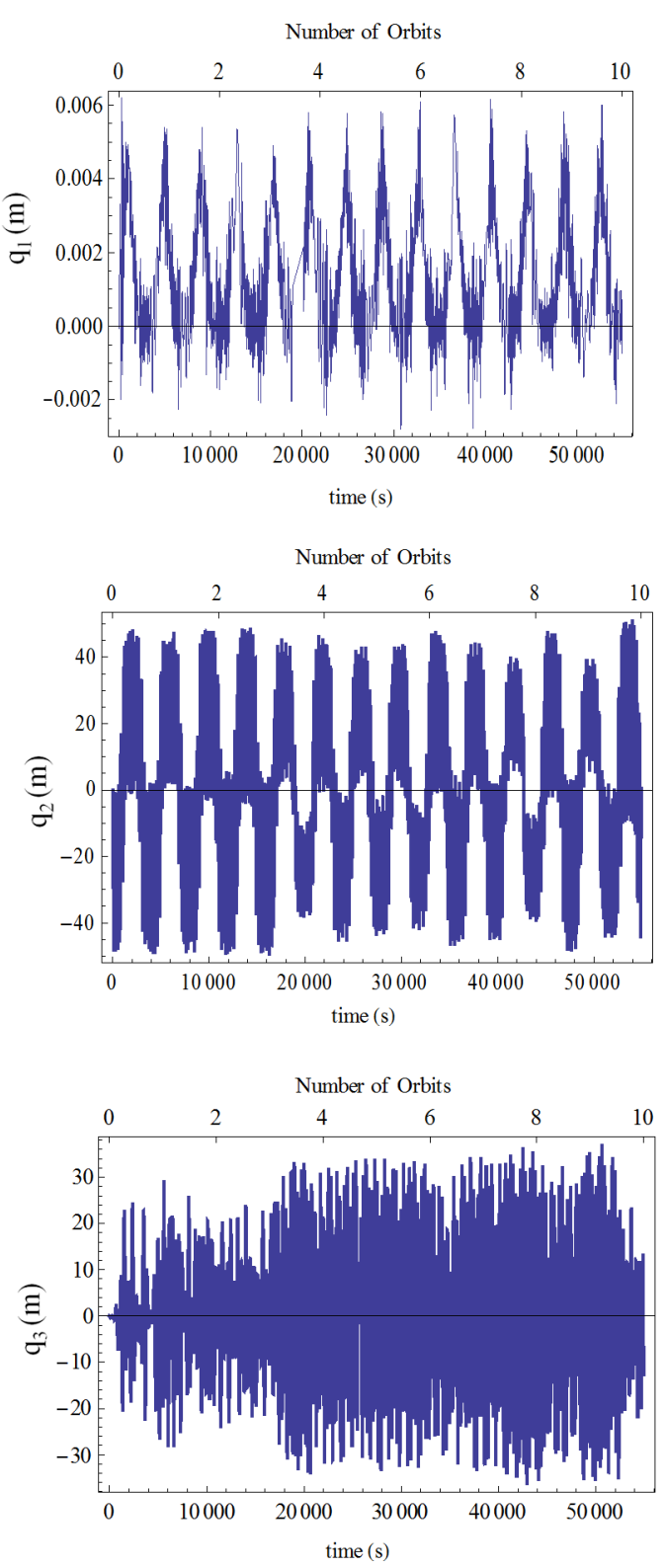

b) with $2.5 \mathrm{MNm}$ torque
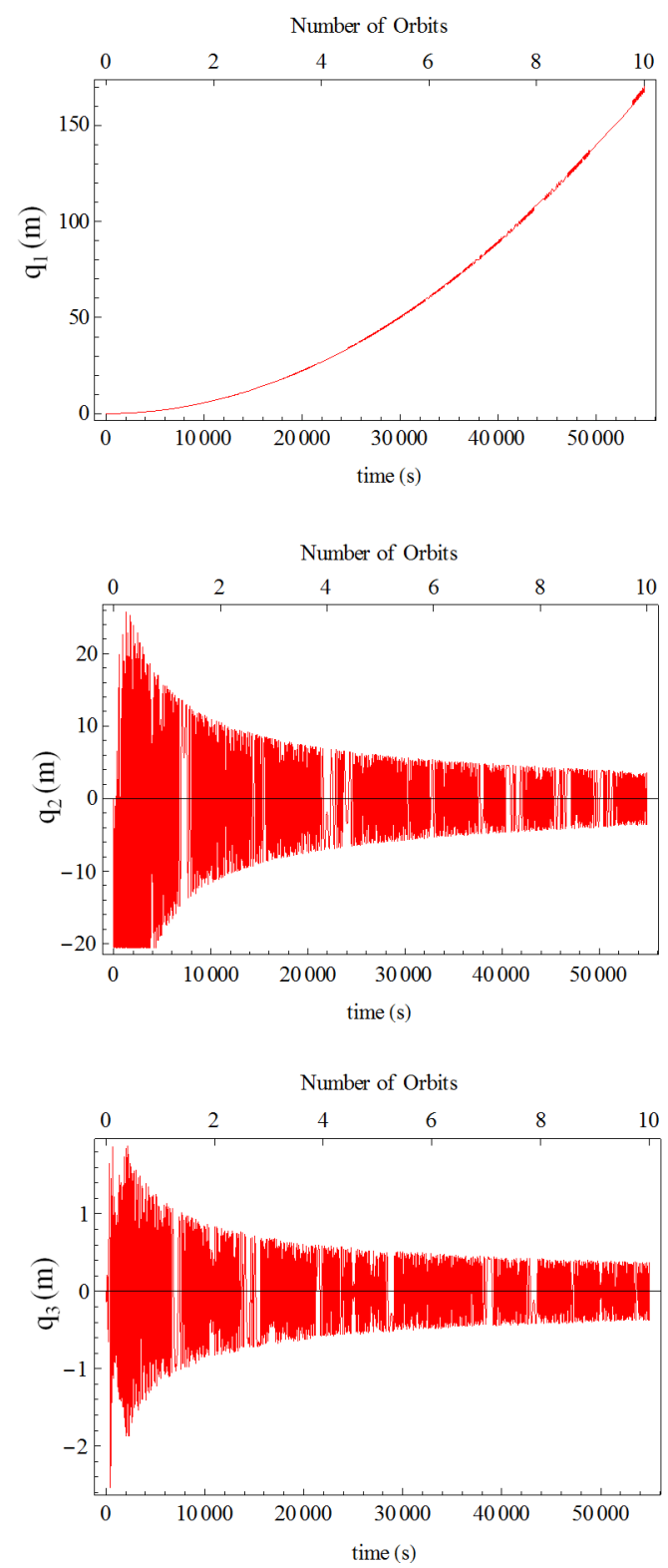

Figure 5: Displacements of the 3D Flexible model of an MMET on a circular orbit. (a) Longitudinal $\left(q_{1}\right)$, lateral $\left(q_{2}\right)$, and transverse (q3) displacement in untorqued condition. (b) Longitudinal $\left(q_{1}\right)$, lateral $\left(q_{2}\right)$ and transverse $\left(q_{3}\right)$ displacement in torqued condition.

The longitudinal displacement in Figure 5(b) appears to show an unbounded exponential growth as compared with the transverse vibration. This phenomenon only occurs when torque is applied to the tether. It can be explained by taking the relationship between the force and the strain for a uniform cross section of a string,

$$
F=E A \varepsilon_{x}
$$


where $\varepsilon_{x}$ is the axial strain and defined by the axial displacement $d u / d x$. In the case of a spinning tether the source of the force comes from the centripetal force. Therefore, by substituting the displacement in the axial direction into equation (16) the relationship between the force and the displacement is given as follows,

$$
F=E A \frac{d u}{d x}
$$

Therefore, when the torque is applied, the centripetal force is increased and for a constant $E$ and tether cross section $A$, the displacement is increased too.

\subsection{Elliptical orbit}

Simulations were carried out for an elliptical orbit with the following orbital elements, $r_{p}=7000000 \mathrm{~m}, e=0.1$

where $r_{p}$ is the perigee of the elliptical orbit, and e is the orbit eccentricity. The tether simulation starts at perigee with initial conditions as in [30],

$\psi(0)=-0.575 \mathrm{rad}, \psi(0)=0 \mathrm{rad} / \mathrm{s}, \alpha(0)=-0.01 \mathrm{rad}, \dot{\alpha}(0)=0 \mathrm{rad} / \mathrm{s}, \theta(0)=0 \mathrm{rad}$

$\dot{\theta}(0)=0.001131 \mathrm{rad} / \mathrm{s}, \gamma(0)=-0.01 \mathrm{rad}, \dot{\gamma}(0)=0 \mathrm{rad} / \mathrm{s}$

The result is shown in Figure 6, with the angular displacements of both tethers being almost identical for the first orbit but then the rigid body model lags behind the flexible model until the sixth orbit. The differences in the angular displacement between both models are clearly shown in Figure 6 (b), where the differences are increasing within the integration time. 


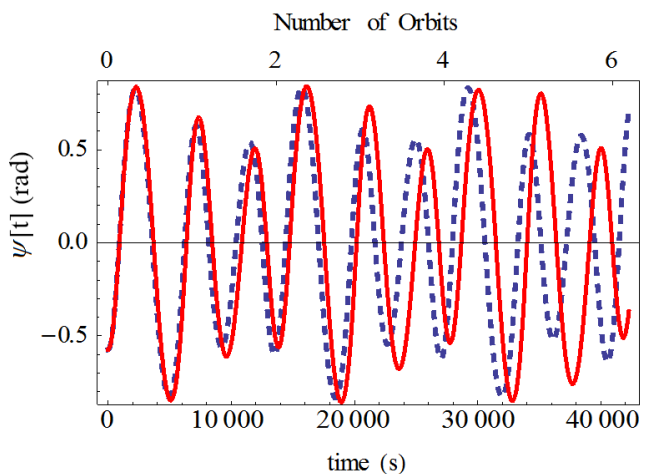

(a)

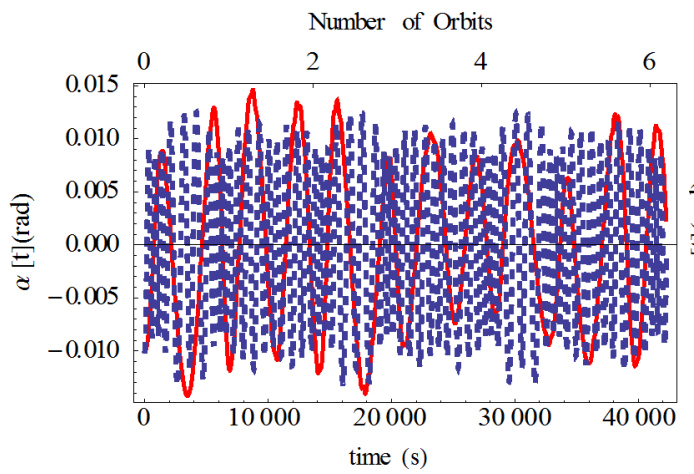

(c)

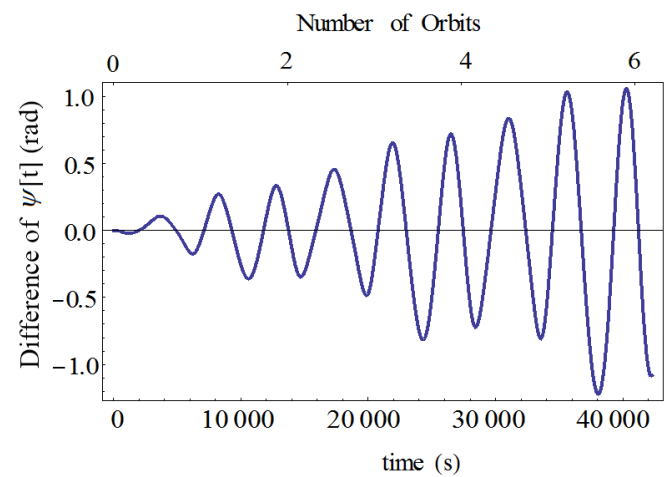

(b)

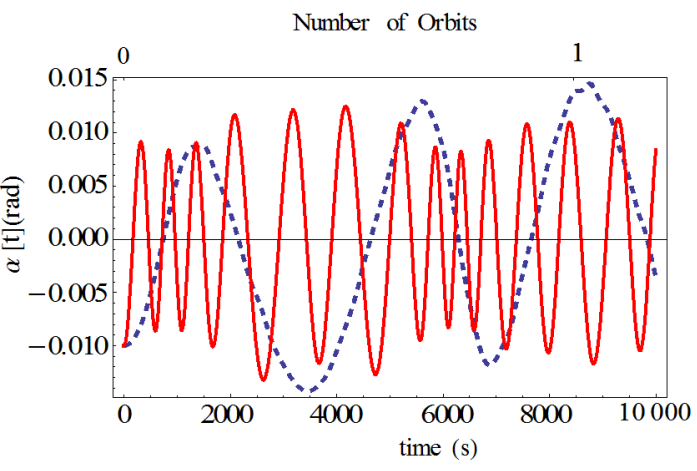

(d)

Figure 6: Responses of rigid body tether (dashed) and flexible tether (line) on an elliptical orbit with zero torque. (a) Angular displacement within 6 orbits , (b) Difference of angular displacement between rigid body tether and flexible tether, (c) Non-planar motions within 6 orbits, and (d) Microview for non-planar motion.

In comparison to the responses for the tether with an applied torque, as shown in Figure 7, the difference in planar motion has shown that the rigid body model moves at a higher rate when compared with the flexible model. But then again, the difference is smaller in comparison to the non-planar motions where the motions in the first orbit show that both models experience decaying motion, with the flexible tether motion decaying at a lower frequency, but with generally higher amplitude. With a longer simulation time the amplitude of the flexible model decreases and is lower than that of the rigid body model, as shown in Figure 7(b). The difference of the orbital radius and true anomaly between the flexible and rigid body motions of the tether in Figure 7(c) and (d) are indistinguishable over a longer period of simulation. It has been shown that a generally very small difference occurs between these two models. This suggests that the flexibility of the tether will make a small alteration to a tether's orbit. 


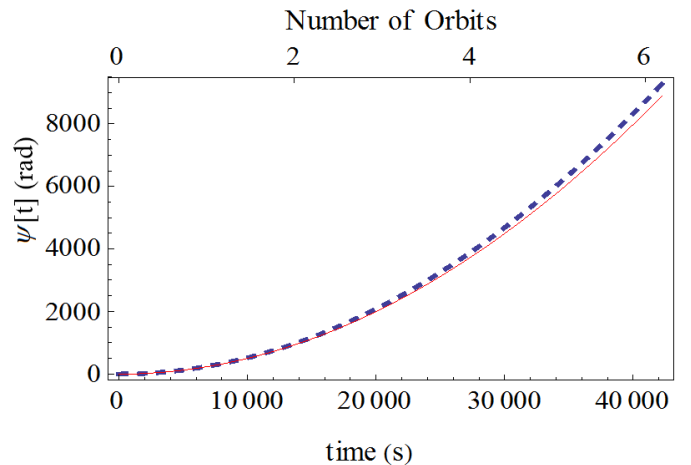

(a)

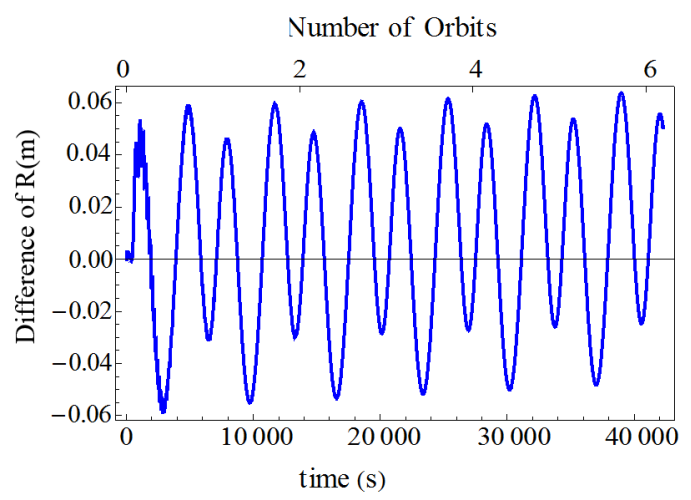

(c)

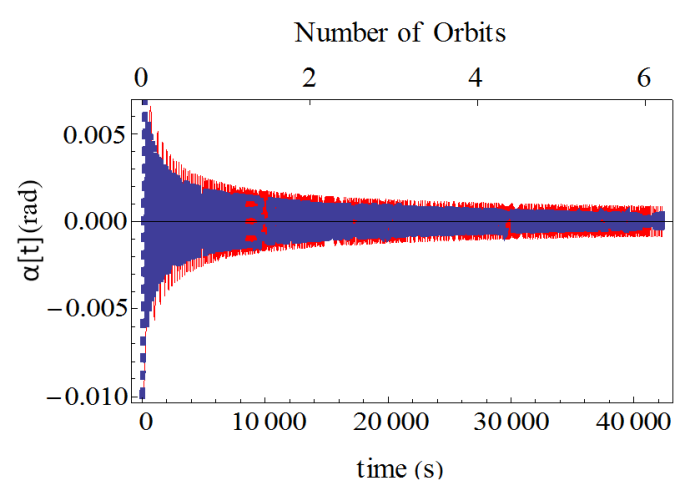

(b)

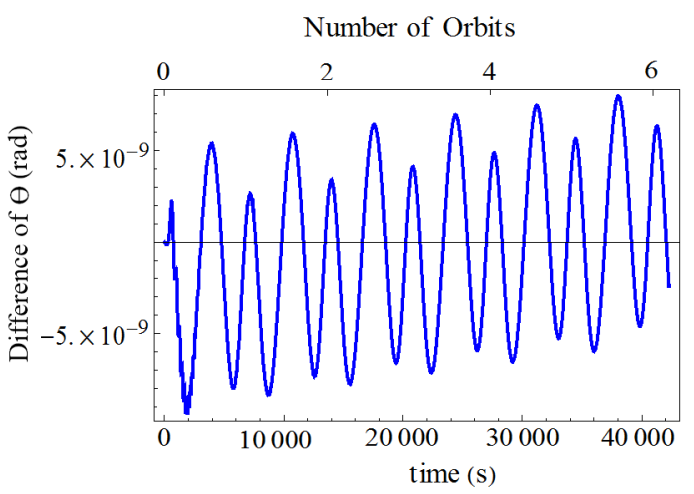

(d)

Figure 7 : (a) and (b) are planar and non-planar motions for3D of a rigid body tether (dashed) and a flexible tether (line), (c) and (d) are the difference in orbital elements between both models on an elliptical orbit with $2.5 \mathrm{MNm}$ torque.

The three dimensional displacement for a tether on an elliptical orbit is shown in Figure 8. The untorqued condition results in the flexible tether oscillating in all directions, with longitudinal, transverse and lateral vibration showing the highest amplitudes of $0.45 \mathrm{~m}, 600 \mathrm{~m}$ and $400 \mathrm{~m}$ for a tether length of $10 \mathrm{~km}$. With the application of torque the displacement in the longitudinal direction increases, but both the transverse and lateral displacements reduce, as shown in Figure 8. 

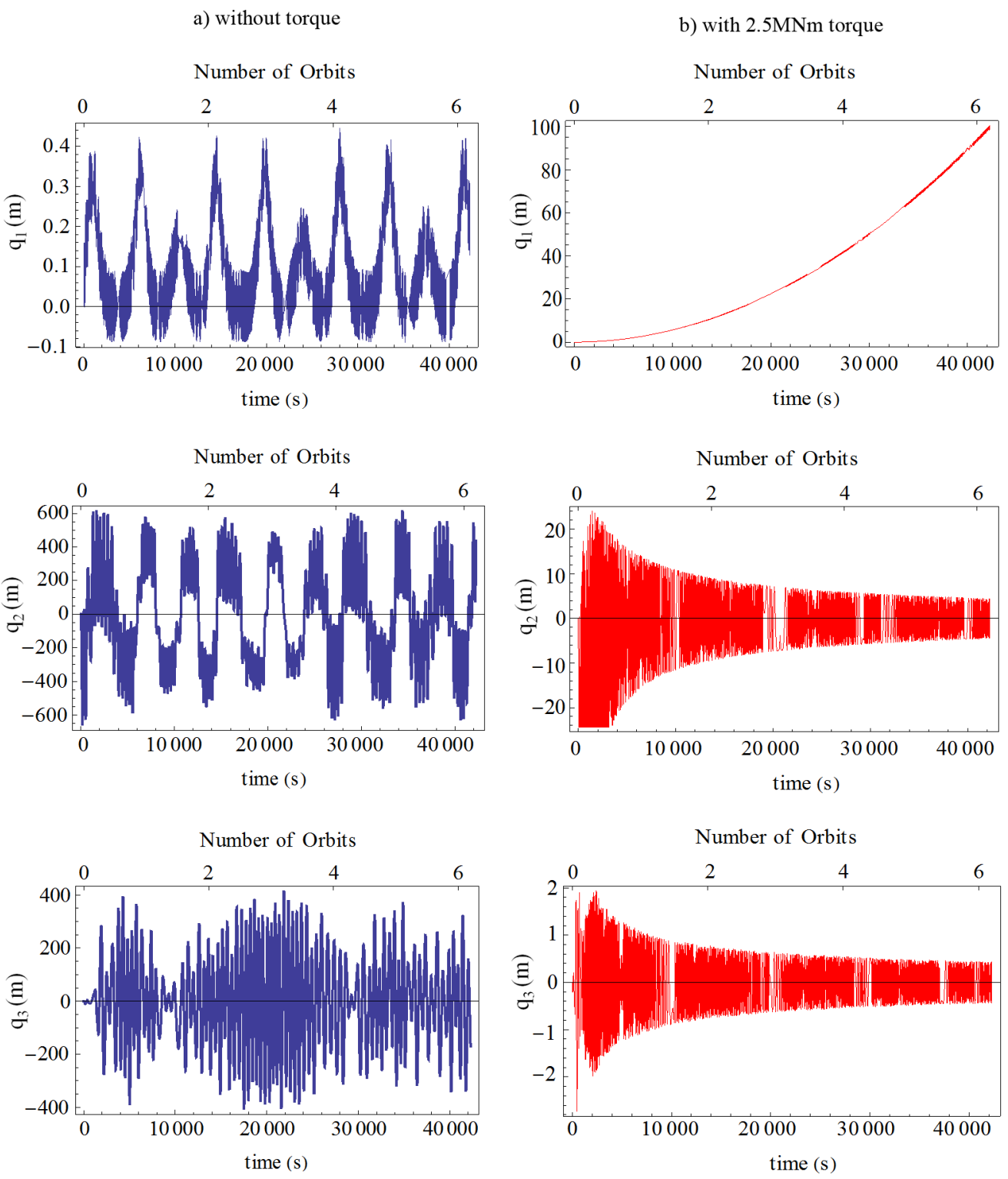

Figure 8 : Displacements of the 3D Flexible model of an MMET on an elliptical orbit. (a) Longitudinal $\left(q_{1}\right)$, lateral $\left(q_{2}\right)$, and transverse $\left(q_{3}\right)$ displacement in untorqued condition. (b) Longitudinal $\left(q_{1}\right)$, lateral $\left(q_{2}\right)$ and transverse $\left(q_{3}\right)$ displacement in torqued condition.

Unlike the unmotorised flexible tether, the application of torque and the effect of centripetal load both cause the longitudinal displacement of the tether to increase significantly within the integration time. Conversely, the transverse vibration has shown a qualitatively different response, in which the vibration decays with time. However this is obviously not a dissipative effect, and in fact this phenomenon is connected to the stiffening effect from the centripetal load experienced by the spinning tether. The centripetal load in the longitudinal direction increases the 
displacement, whilst the lateral stiffening effect reduces the amplitude of vibration in the transverse and lateral directions.

\subsection{Comparison between the 2D and 3D Flexible Models.}

The difference of the responses between two dimensional (2D) and three dimensional (3D) motions of the flexible model are shown in Figures 9. The derivation of equations of motion for the $2 \mathrm{D}$ flexible model has been presented in [33]. Simulating the differences in angular displacement and angular velocities between these two models shows that a difference occurs and even though it is relatively small, it is still significant for the global motion of the tether. The existence of the non-planar variable $(\alpha)$ in the equations of motion of the 3D model alters the orbit of the tether, but at a smaller scale. It is shown, in Figure 9 (c) that the maximum difference in the magnitude $\mathrm{R}$ of the position vector, within the simulation time is $0.0014 \mathrm{~m}$ and the difference in the true anomaly is insignificant and within the range of $8 \times 10^{-11} \mathrm{rad}$, as shown in Figure 9 (d).

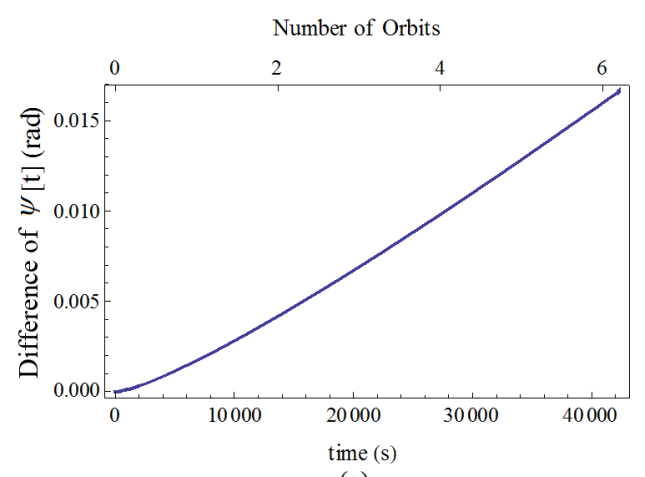

(a)

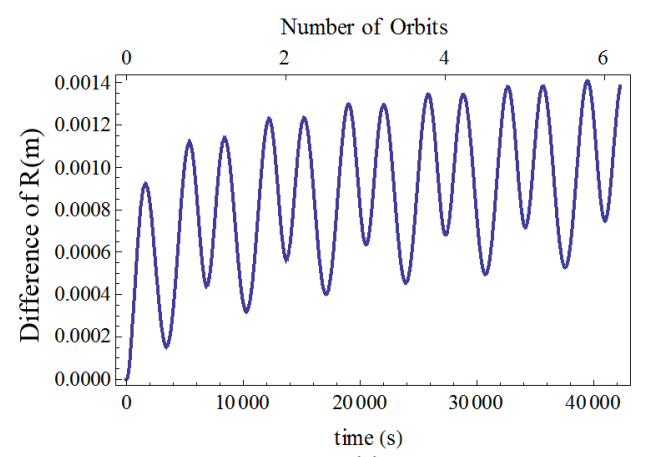

(c)

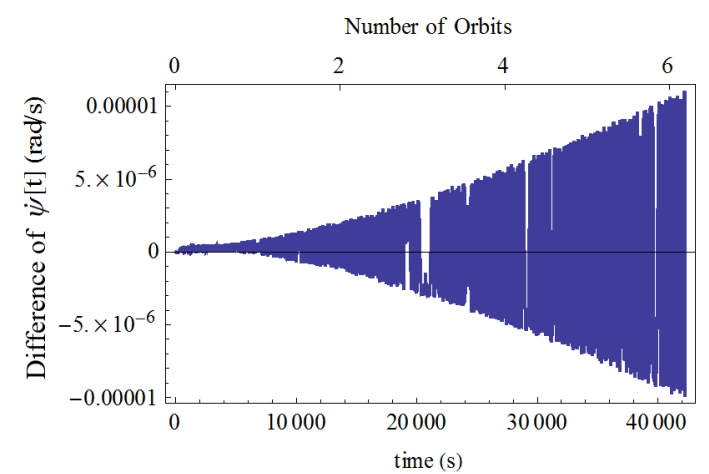

(b)

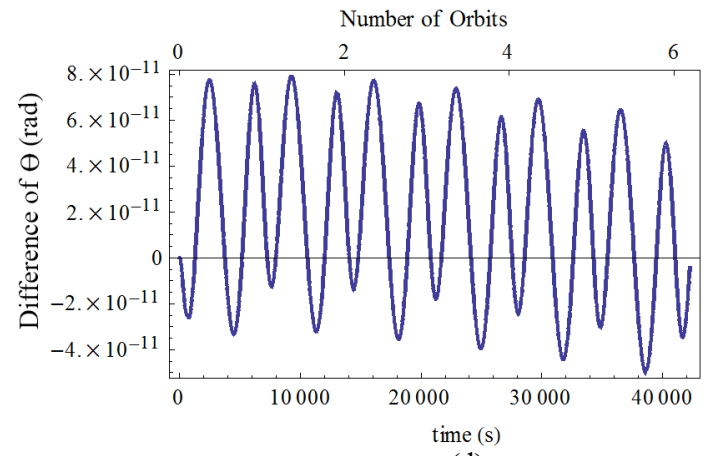

(d)

Figure 9: The difference between: (a) angular displacement, (b) angular velocity, (c) radius, and (d) true anomaly, for the 2D and 3D flexible tether model. 
The local displacement of the tether, Figure 10, shows that both models are displaying the same trend, where the longitudinal displacement is increasing and the transverse displacement is decaying, due to the reason explained in section 6.1, with an increase in simulation time as required by the inclusion of the stiffening effect caused by the centripetal force.

(e)

Number of Orbits

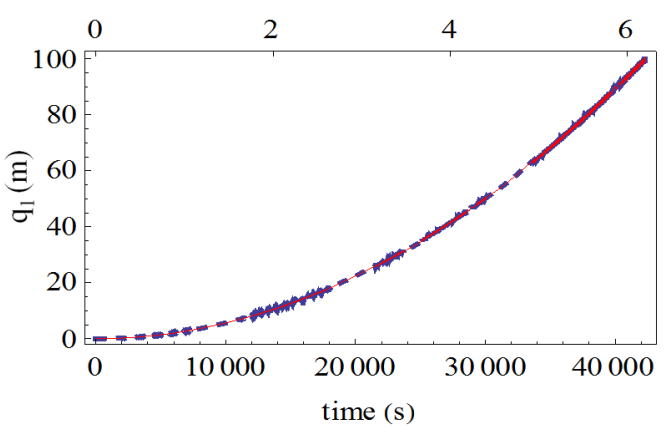

(f)

Number of Orbits

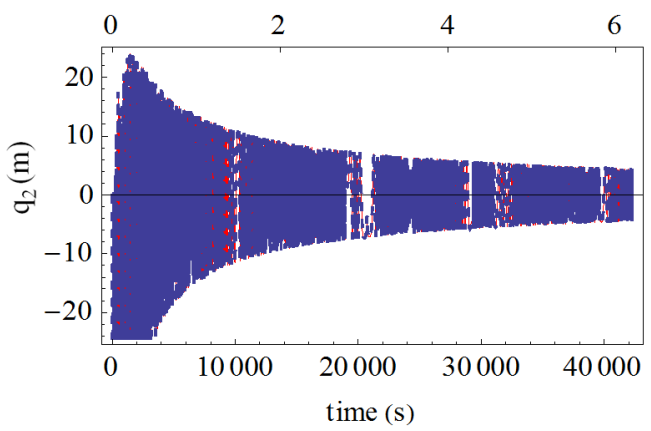

Figure 10: (e) longitudinal displacement $\mathrm{q}_{l}[\mathrm{t}]$ and (f) transverse displacement $q_{2}[\mathrm{t}]$ of the $2 \mathrm{D}$ and 3D flexible tether models.

\section{Equations of Motions for Dynamical System Analysis}

Ziegler [7] transformed the equations of motion of an MMET by expressing the dependent variables as a function of the orbital true anomaly, on the assumption that the tether remains in a Keplerian orbit. This transformation method has been applied to this new flexible model of the MMET. Based on that the derived equations of motion for the in-plane angle of the two dimensional flexible model in [33], and the axial and transverse displacements with respect to the true anomaly, are given as, 


$$
\begin{aligned}
& \frac{M_{P} L R \mu \sin \psi}{\left(L^{2}+R^{2}+2 L R \cos \psi\right)^{3 / 2}}+-\frac{M_{P} L R \mu \sin \psi}{\left(L^{2}-R^{2}+2 L R \cos \psi\right)^{3 / 2}}+\left(\frac{5}{6} \rho A L^{3}+\frac{4}{\pi} \rho A L^{2} q_{1}\right. \\
& \left.\rho A L q_{1}^{2}+\rho A L q_{2}^{2}+2 M_{P} L^{2}+\frac{1}{2} M_{m} r_{m}^{2}+M_{p} r_{p}^{2}+\frac{1}{2} \rho A L r_{T}^{2}\right)\left(\left(\dot{\theta} \dot{\theta}^{\prime}\right)+\left(\dot{\theta} \dot{\theta}^{\prime} \psi^{\prime \prime}+\dot{\theta}^{2} \psi^{\prime \prime}\right)\right) \\
& +\left(\frac{4}{\pi} \rho A L^{2} q_{1}+2 \rho A L\left(q_{1} q_{1}^{\prime}+q_{2} q_{2}^{\prime}\right)+\frac{4}{\pi} \rho A L^{2} \dot{\theta}^{2} q_{1} \psi^{\prime}+2 \rho A L\left(q_{1} q_{1}^{\prime}+q_{2} q_{2}^{\prime}\right) \psi^{\prime}\right) \dot{\theta}^{2} \\
& -\rho A L q_{2}\left(\dot{\theta} \dot{\theta}^{\prime} q_{1}^{\prime}+\dot{\theta}^{2} q_{1}^{\prime \prime}\right)+\left(\frac{2}{\pi} \rho A L^{2}+\rho A L q_{1}\right)\left(\dot{\theta} \dot{\theta}^{\prime} q_{2}^{\prime \prime}+\dot{\theta}^{2} q_{2}^{\prime \prime}\right)+ \\
& -\sum_{i=1}^{N}-\frac{(2 i-1) \mu \rho A L^{2} R \sin \psi}{2 N^{2}\left(R^{2}+\frac{(2 i-1)^{2} L^{2}}{4 N^{2}}-\frac{(2 i-1) R L}{N} \cos \psi\right)^{3 / 2}} \\
& -\sum_{i=1}^{N} \frac{(2 i-1) \mu \rho A L^{2} R \sin \psi}{2 N^{2}\left(R^{2}+\frac{(2 i-1)^{2} L^{2}}{4 N^{2}}-\frac{(2 i-1) R L}{N} \cos \psi\right)^{3 / 2}}=\tau
\end{aligned}
$$

$$
\begin{aligned}
& \rho A L\left(\dot{\theta} \dot{\theta}^{\prime} q_{1}^{\prime}+\dot{\theta}^{2} q_{1}^{\prime \prime}\right)+\frac{A E_{o} \pi^{2}}{L} q_{1}+\frac{15}{8 L^{3}} T_{o} \pi^{4} q_{1}^{3}+\left(\frac{3}{4 L^{3}} \pi^{4}\left(T_{o}-A E_{o}\right)\right) q_{1} q_{2}^{2}- \\
& \rho A L q_{1}\left(\dot{\theta}^{2}-2 \dot{\theta}^{2} \psi^{\prime}\right)-\left(\rho A L q_{1}+\frac{2 \rho A L^{2}}{\pi}\right)\left(\dot{\theta}^{2} \psi^{\prime \prime 2}\right)-\rho A L\left(2 q_{2}^{\prime} \dot{\theta}^{2}+q_{2} \dot{\theta} \dot{\theta}^{\prime}\right)- \\
& \left(\frac{4 \rho A L^{2}}{\pi}-2 \rho A L q_{2}^{\prime}\right) \psi^{\prime} \dot{\theta}^{2}-\rho A L q_{2}\left(\dot{\theta} \dot{\theta}^{\prime} \psi^{\prime}+\dot{\theta}^{2} \psi^{\prime \prime}\right)=0 \\
& \rho A L\left(\dot{\theta} \dot{\theta}^{\prime} q_{2}^{\prime}+\dot{\theta}^{2} q_{2}^{\prime \prime}\right)+\frac{T_{o} \pi^{2}}{L} q_{2}+\left(\frac{3}{8 L^{3}} \pi^{4}\left(A E_{o}-T_{o}\right)\right) q_{2}^{3}-\rho A L q_{2}\left(\dot{\theta}^{2}+2 \dot{\theta}^{2} \psi^{\prime \prime}\right) \\
& -\rho A L q_{2} \dot{\theta}^{2} \psi^{\prime 2}+\left(\frac{3}{4 L^{3}} \pi^{4}\left(T_{o}-A E_{o}\right)\right) q_{1}^{2} q_{2}+\left(\frac{2 \rho A L^{2}}{\pi}+\rho A L q_{1}\right) \dot{\theta} \dot{\theta}^{\prime} \\
& +2 \rho A L q_{1}^{\prime}\left(\dot{\theta}+\dot{\theta} \psi^{\prime}\right)+\left(\frac{2 \rho A L^{2}}{\pi}+\rho A L q_{1}\right)\left(\dot{\theta} \dot{\theta}^{\prime} \psi^{\prime}+\dot{\theta}^{2} \psi^{\prime \prime}\right)=0
\end{aligned}
$$

These equations of motion, given in terms of the true anomaly, are used for further dynamical analysis of the two dimensional flexible tether in the next section. 


\subsection{Transition from Regular to Chaotic Motion for Two Dimensional Flexible Tether}

Dynamical systems sometimes enter regions of apparently irregular behaviour, making predictions of their future dynamics extremely difficult, particularly if the system appears to have been sensitive to the initial conditions. In this study, the initial conditions have the potential to influence the motion of the tether in $\psi$, and also in $\alpha$ for the three dimensional case. A change in these initial conditions can lead to irregularities in the trajectories in those variables and these are seen when they are depicted in a bifurcation diagram or on a Poincaré map. Chaotic behaviour has been evident in previous models of the motorised tether [7, 30] and in such cases modifications to various tether parameters can potentially be used to control the motion of the system [37]. Figure 11 shows the motion of a flexible tether entering the chaotic region for orbit eccentricities greater than 0.28 . This is indicated by the dispersed points for $e>0.28$.

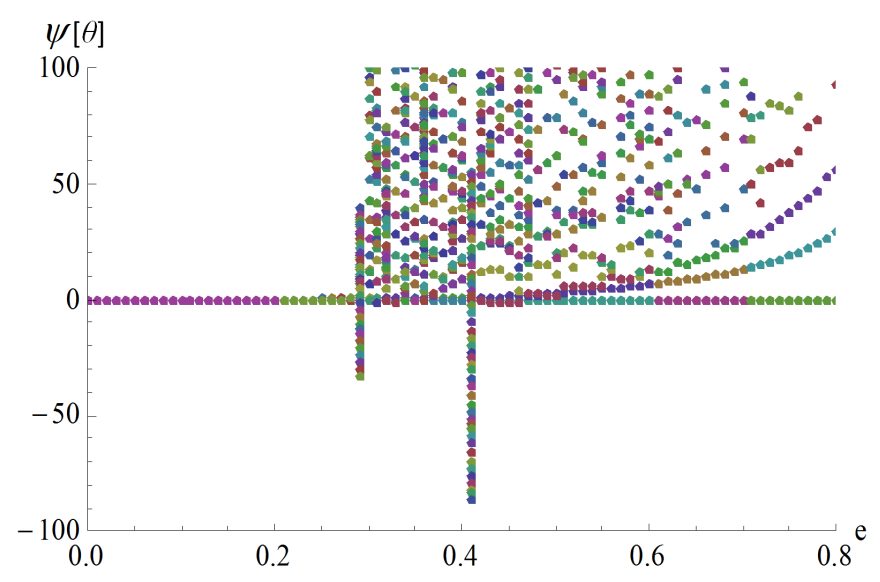

Figure 11: Bifurcation Diagram of the angular displacement with respect to the orbit eccentricity with initial conditions $\psi(0)=0 \mathrm{rad}$, and $\dot{\psi}(0)=0 \mathrm{rad} / \mathrm{s}$ and a step size of $e=0.01$.

The region between $0<e<0.3$ has been magnified in Figure 12 and shows periodic windows and bands of points that represent the behaviour of the system both in regular and chaotic motion. In Figure 12 the system is clearly seen to start what appears to be chaotic motion at $e=0.28$. Period three motion is also visually distinguishable within the regular motion region. The bifurcation diagram for the 
flexible model is compared with the bifurcation diagram for the rigid body model in Figure 13.

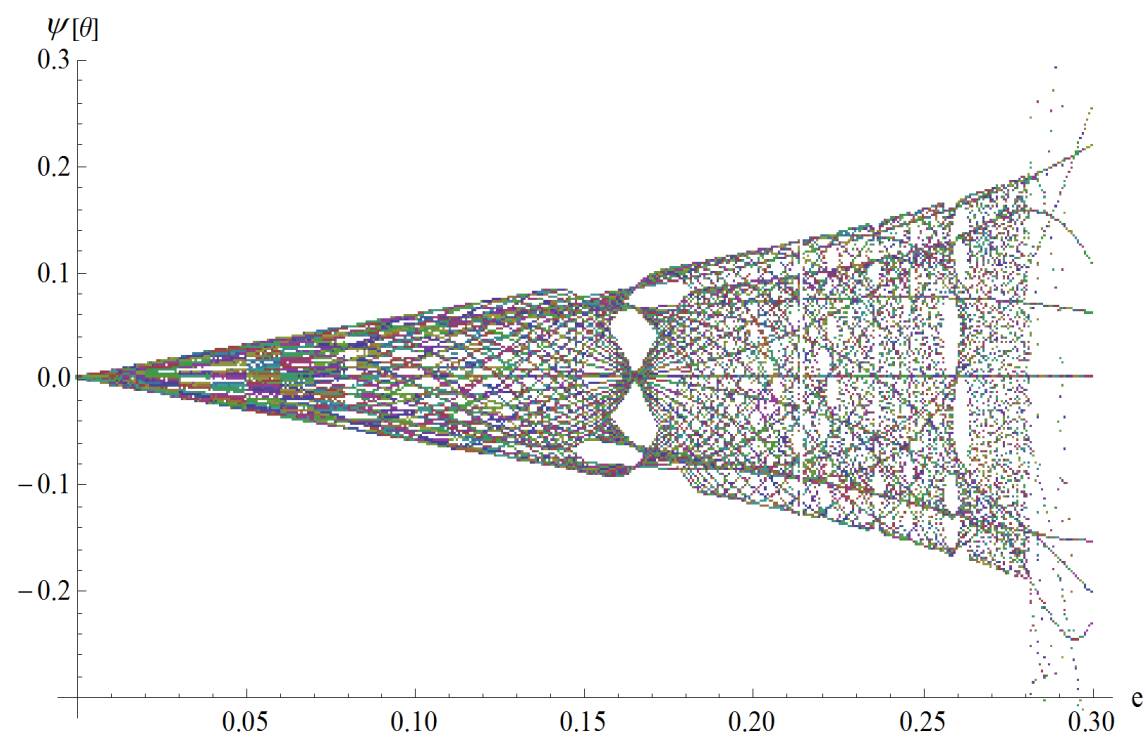

Figure 12: Bifurcation Diagram of the angular displacement of the flexible model with respect to the orbit eccentricity with initial conditions $\psi(0)=0 \mathrm{rad}$, and $\dot{\psi}(0)=0 \mathrm{rad} / \mathrm{s}$ and a step size of $e=0.0005$.

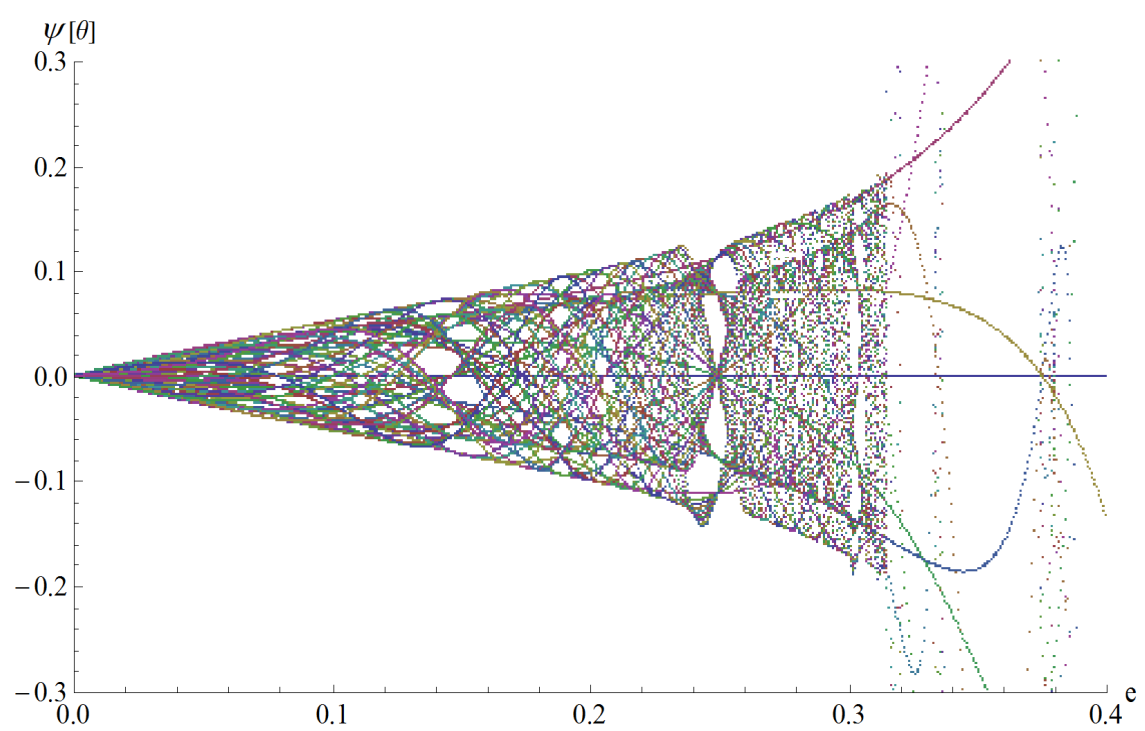

Figure 13 : Bifurcation Diagram of the angular displacement of the rigid body model with respect to the orbit eccentricity with initial conditions $\psi(0)=0 \mathrm{rad}$, and $\dot{\psi}(0)=0 \mathrm{rad} / \mathrm{s}$ and a step size of $\mathrm{e}=$ 0.0005 . 
Both figures basically agree with the finding by Karasopoulos and Richardson [38], Fujii and Ichiki [39] and Ziegler [30], where Fujii and Ichiki [39] found that chaotic motion occurred approximately at $e>0.280$ for an elastic tether with a longitudinal flexibility of $104 \mathrm{~N} / \mathrm{m}$, and Karasopoulos and Richardson [38] and Ziegler [30] showed that the rigid body tether should start to spin up at $e>0.314$. The initial state of the bifurcation diagram for the rigid body tether is a period one per orbit, but on sampling the point at $e=0$ for the flexible model the Poincare map in Figure 14 shows that the flexible model does not display period one motion, but suggests that the motion has crossed the zero point for quite a number of orbits.
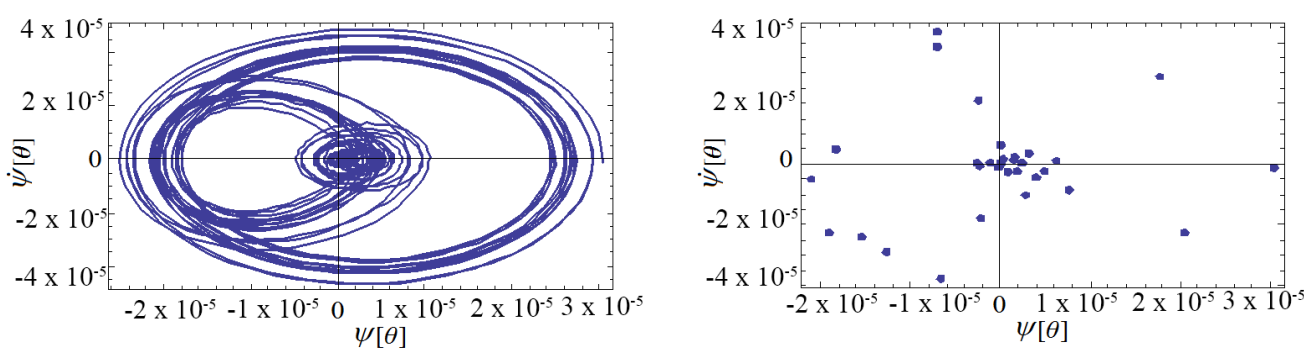

Figure 14: Phase portrait and Poincaré Map for flexible tether motion at $\mathrm{e}=0$ with initial conditions $\psi(0)=0 \mathrm{rad}$, and $\dot{\psi}(0)=0 \mathrm{rad} / \mathrm{s}$

In making a comparison between Figures 12 and 13 period three motion occurs in different regions, whereby period three motion of the flexible tether is approximately at $e=0.165$ and for the rigid body model it is at 0.280 . Integrating equations (18) and (19) for 200 orbits leads to Figure 15 representing the Poincaré map for period three motion of the flexible tether.

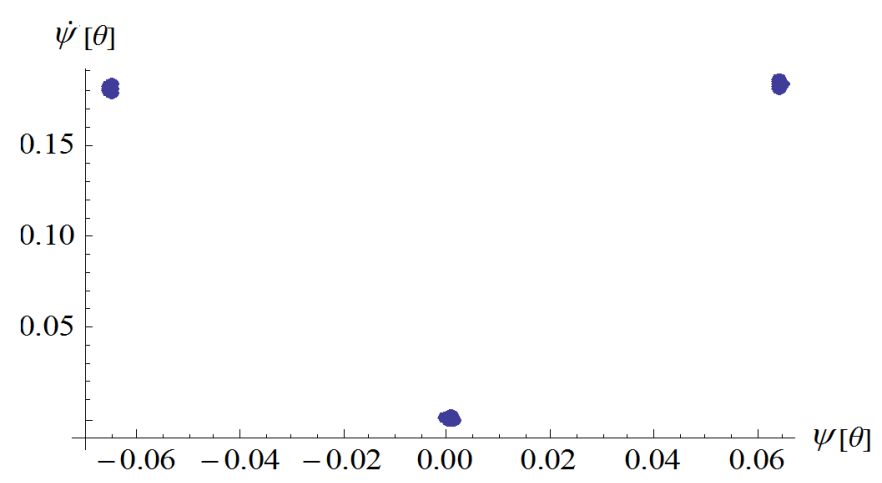

Figure 15 : Poincaré map for the flexible tether, sampling at each perigee crossing for 200 orbits with e $=0.1654$ 
On sampling the points for 200 orbits of the rigid body model the Poincare map shows that the tether is displaying period three motion, but the precise position is drifting quasi-periodically, as shown in Figure 16.

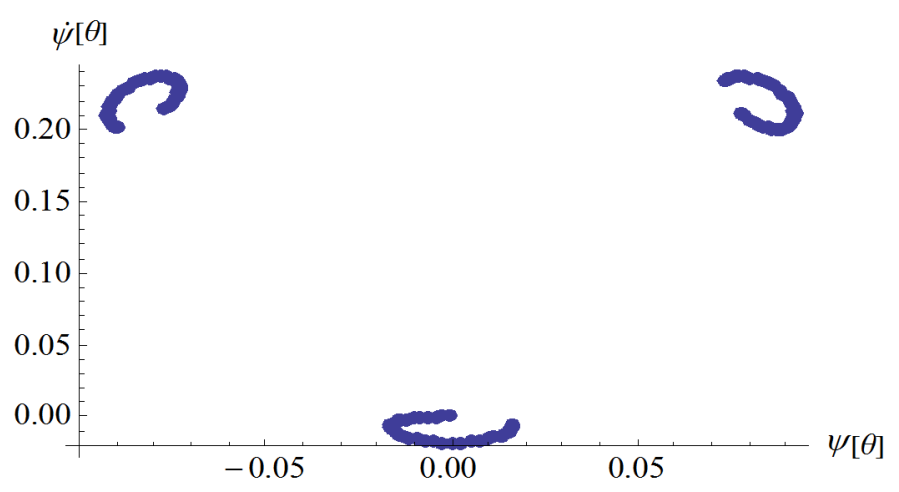

Figure 16: Poincaré map for the rigid body tether, sampling at each perigee crossing for 200 orbits with $e=0.2479$

Then, on sampling the specific point at $e=0.05$ for 200 orbits, as in Figure 17, it is shown that the motion is stable and periodic.

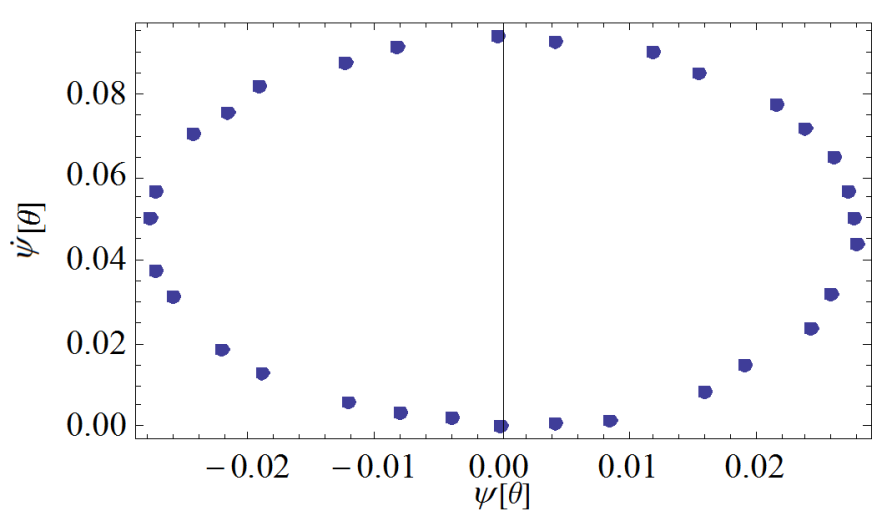

Figure 17: Poincaré map for the flexible tether, sampling at each perigee crossing for 200 orbits with $e$ $=0.05$

Motion of period 5 appears for $e=0.26$ for the flexible tether, as shown in Figure 18 for the sample of points over 30 orbits. By integrating equations (18) and (19) for a longer period Figure 19 shows the same phenomenon as seen in Figure 16, in which the tether's position is drifting quasi-periodically. Therefore, it is suggested here that the lower sampling period may well mislead the prediction of tether motion in the longer term. 


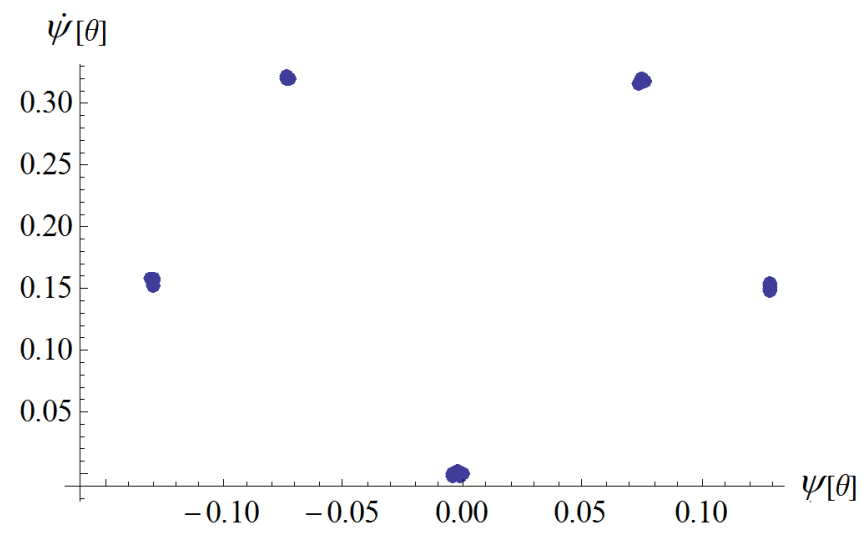

Figure 18 : Poincaré map for the flexible tether, sampling at each perigee crossing for 30 orbits with $e$ $=0.26$

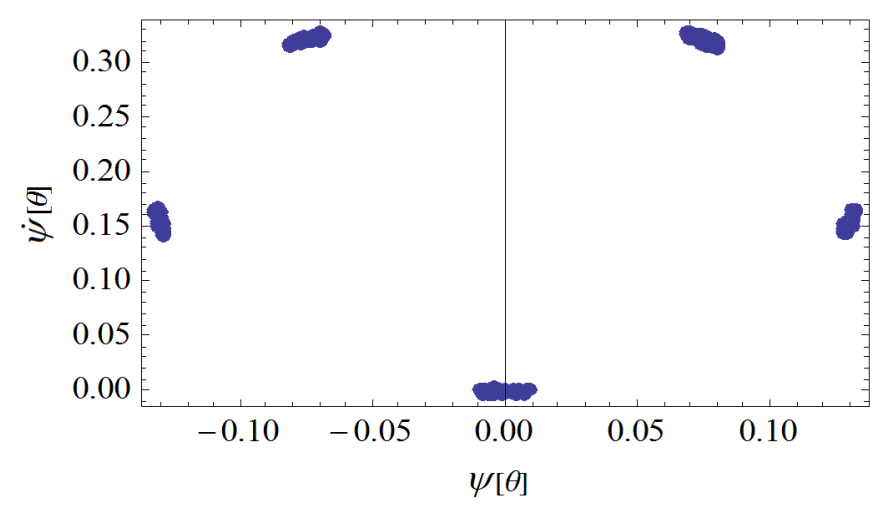

Figure 19 : Poincaré map for the flexible tether, sampling at each perigee crossing for 150 orbits with $e$ $=0.26$

When integrating the equations of motion for the rigid body tether with a similar eccentricity and initial conditions, the rigid body tether shows different dynamic conditions when integrated over 150 orbits. Quasi-periodic motion has appeared, depicted by the closed curve seen in the Poincaré map in Figure 20, and it is shown here that the flexibility of the tether is strongly influencing the tether's global motion. 


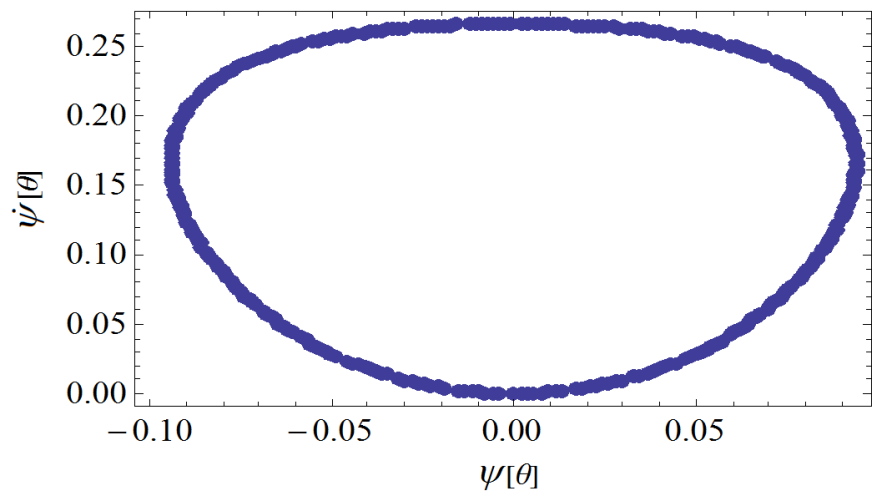

Figure 20 : Poincaré map for the rigid body tether, sampling at each perigee crossing for 150 orbits with $e=0.26$

In the case of initial conditions for which $\psi(0)=0.5 \mathrm{rad}$ and $\psi(0)=0 \mathrm{rad} / \mathrm{s}$, the bifurcation diagrams for the flexible and rigid body tethers can be seen in Figures 21 and 22.

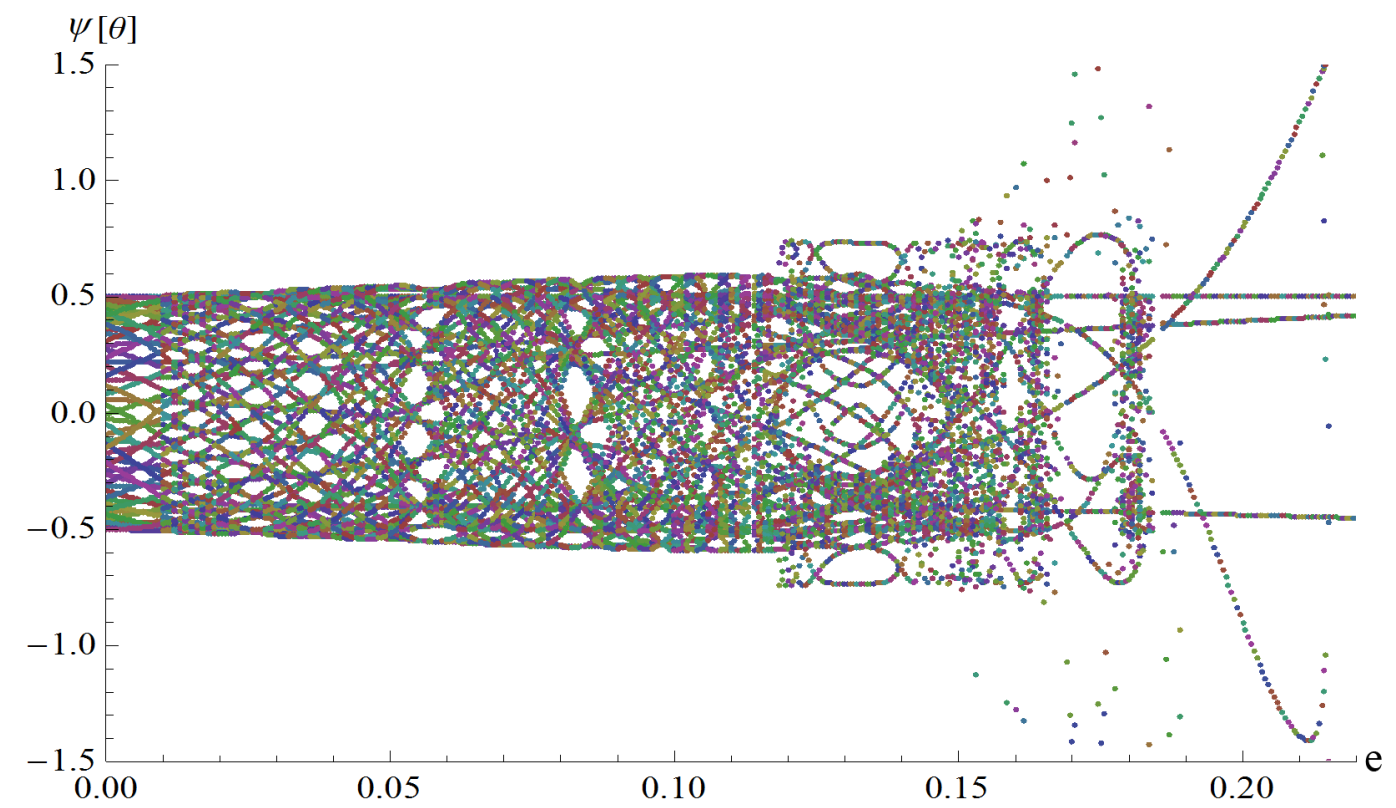

Figure 21: Bifurcation Diagram of the angular displacement of the flexible model with respect to the orbit eccentricity with initial conditions $\psi(0)=0.5 \mathrm{rad}$, and $\psi(0)=0 \mathrm{rad} / \mathrm{s}$ and a step size of $e=$ 0.0005 . 


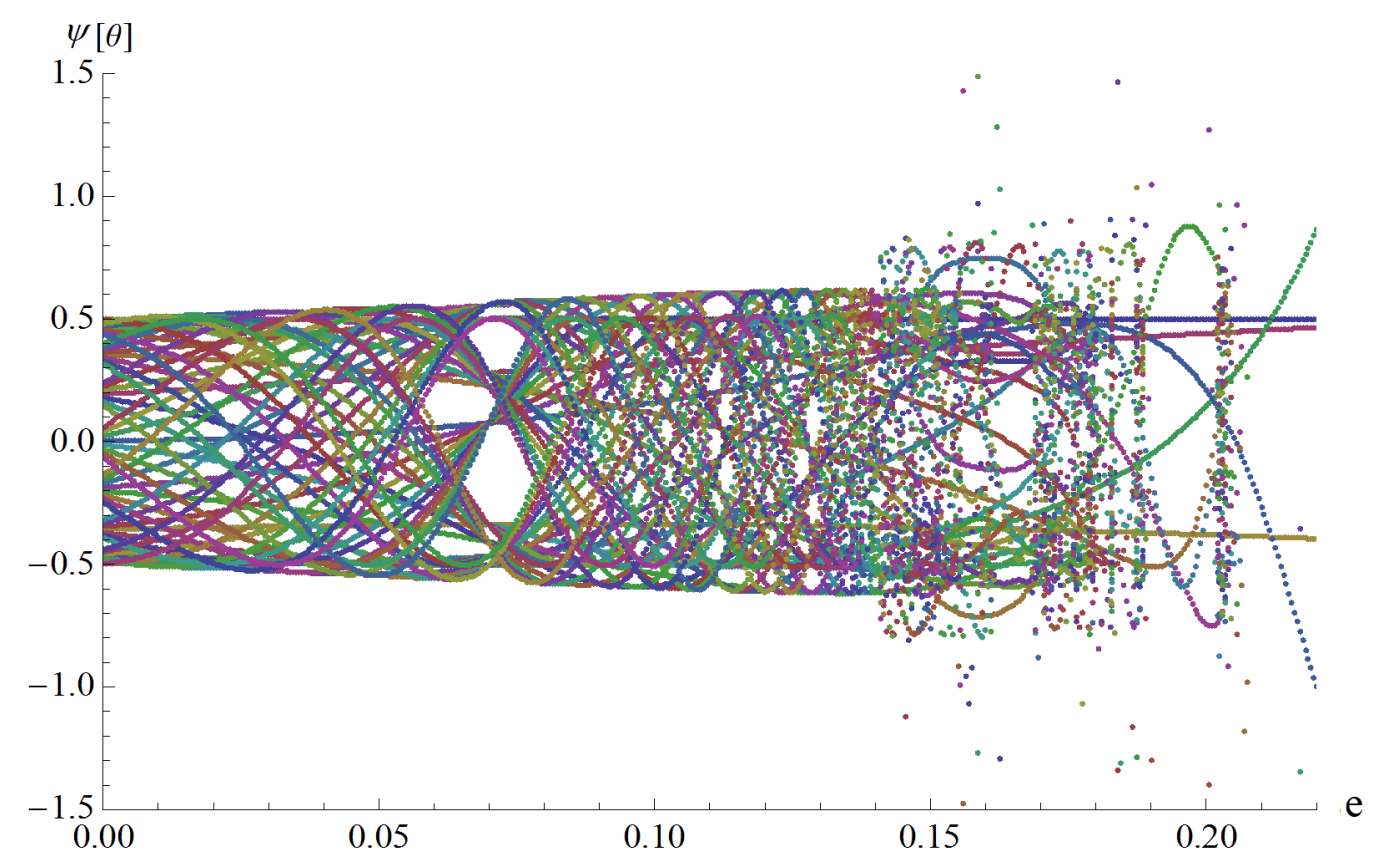

Figure 22: Bifurcation Diagram of the angular displacement of the rigid body model with respect to the orbit eccentricity with initial conditions $\psi(0)=0.5 \mathrm{rad}$, and $\dot{\psi}(0)=0 \mathrm{rad} / \mathrm{s}$ and a step size of $e=$ 0.0005 .

The points at which the tether commences to visit all regions reduce from $e=0.28$ to $\mathrm{e}=0.11$ and it can be seen that the initial angular velocity has a significant influence on the start of the chaotic motion. In comparison between the flexible and rigid body models, the region of chaos starts at $e=0.14$ for the rigid body tether. Consequently, the flexibility of the tether is seen, in addition to the eccentricity and initial conditions, to have an influence on the onset of chaos.

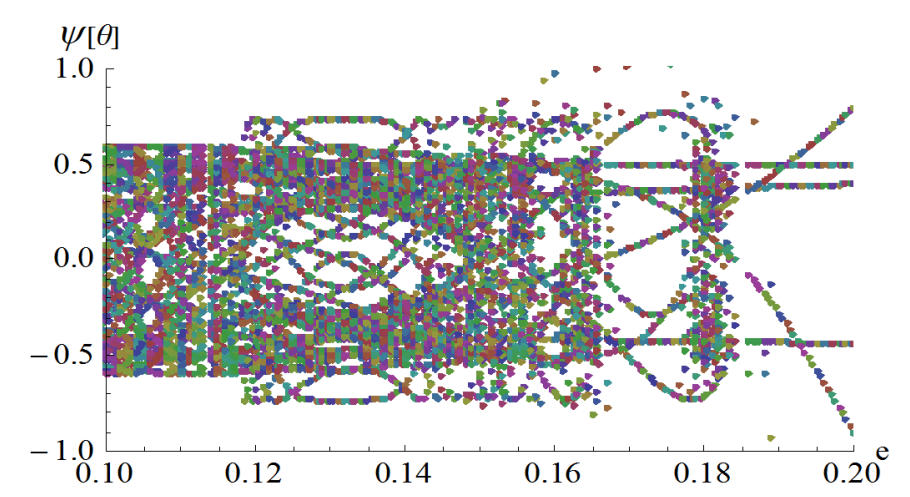

Figure 23: Bifurcation Diagram of the angular displacement of the flexible model with respect to the orbit eccentricity between $0.1 \leq \mathrm{e} \leq 0.2$ with initial conditions $\psi(0)=-0.5 \mathrm{rad}$, and $\dot{\psi}(0)=0 \mathrm{rad} / \mathrm{s}$ for a step size of $e=0.0005$. 

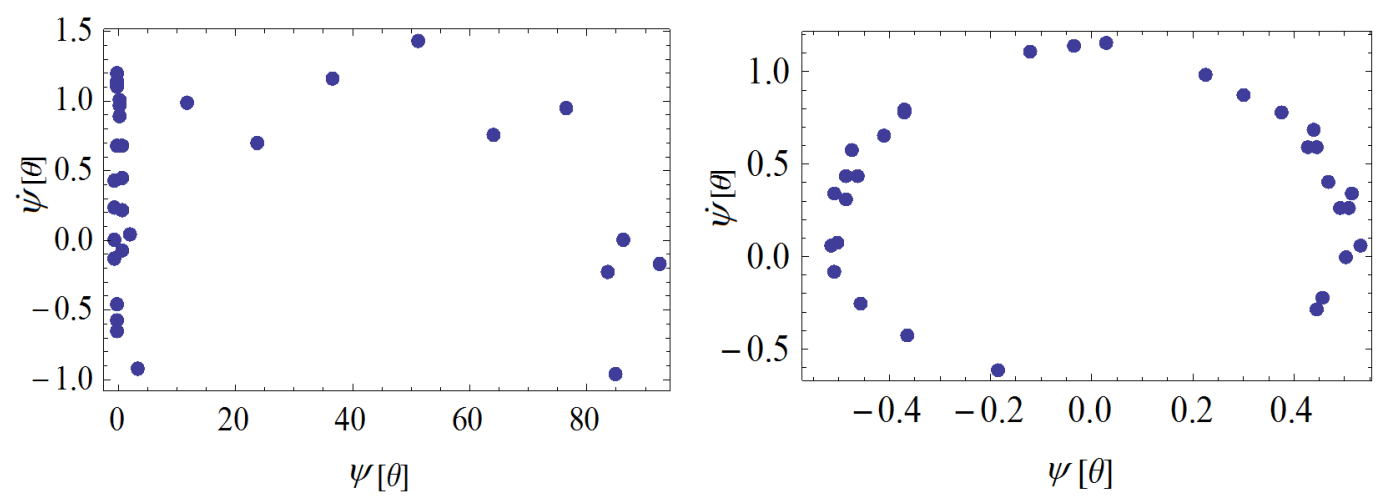

Figure 24 : Poincaré maps for the flexible tether with initial condition (a) $\psi(0)=-0.5 \mathrm{rad}$ and (b) $\psi(0)$ $=0.5 \mathrm{rad}$ at $e=0.15$ for 30 orbits.

The initial conditions are then changed to $\psi(0)=-0.5 \mathrm{rad}$ and $\dot{\psi}(0)=0 \mathrm{rad} / \mathrm{s}$ to observe the motion of the tether with negative initial conditions, and the bifurcation diagram for this is given in Figure 23. In general, the bifurcation diagram in Figure 23 is seen to have a rather similar shape to that of Figure 21. However, the difference can be seen from the region where the chaos just starts to begin at approximately $e \approx 0.12$. The diagram shows the points in Figure 21 and 23 dispersed in different trajectories when entering the chaotic region.

Figure 24 sampling the points with the same eccentricity to show the difference motion between the different initial conditions.

\subsection{Route to Chaos for a Three Dimensional Flexible Tether.}

The non-planar motion is more computationally complex still and longer computing times are required. Therefore the dynamical analysis for the three dimensional model of the flexible tether is limited to the route to chaos. Figure 25 shows the bifurcation diagram for the nonplanar motion of the flexible tether with initial conditions $\psi(0)=0 \mathrm{rad}, \dot{\psi}(0)=0 \mathrm{rad} / \mathrm{s}$, and $\alpha(0)=0.1 \mathrm{rad}$ for $0.1 \leq e \leq 0.3$. From Figure 25 , chaos is found, starting approximately at $e \approx 0.28$ in which it is similar in 
form to the planar motion of Figure 12. This agrees with Figure 7 previously where the initial displacement of $\alpha$ does not significantly influence the planar motion of the flexible tether with the initial condition $\psi(0)=0 \mathrm{rad}$.

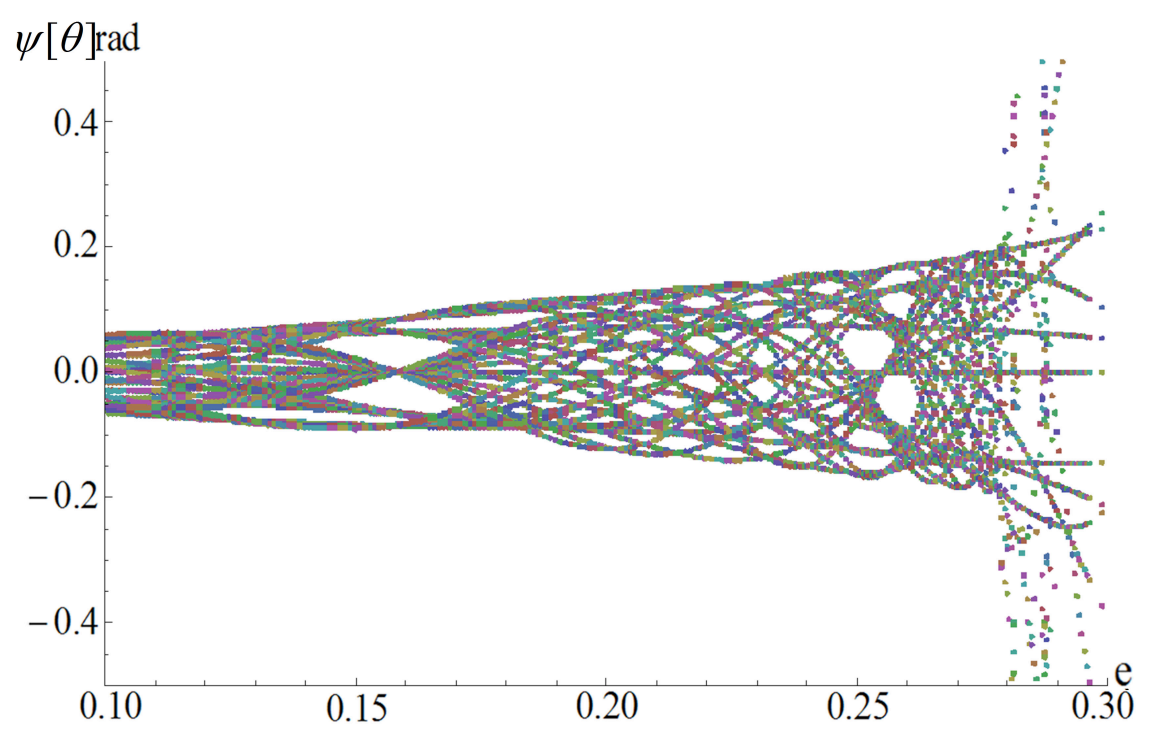

Figure 25 : Bifurcation Diagram of the angular displacement of the flexible model with respect to the orbit eccentricity with initial conditions $\psi(0)=0 \mathrm{rad}, \dot{\psi}(0)=0 \mathrm{rad} / \mathrm{s}, \alpha(0)=0.1 \mathrm{rad}$ and a step size of $e$ $=0.00075$.

In comparison with the three dimensional motion of the rigid tether, Figure 26 samples the point at $e=0.15, \psi(0)=0 \mathrm{rad}$ and $\dot{\psi}(0)=0 \mathrm{rad} / \mathrm{s}$ for both models and the results evidently show the Poincaré Map of the flexible model does not display the same motion as the rigid body. This again shows that the flexibility of the tether has a significant impact on the global motion. 
(a)

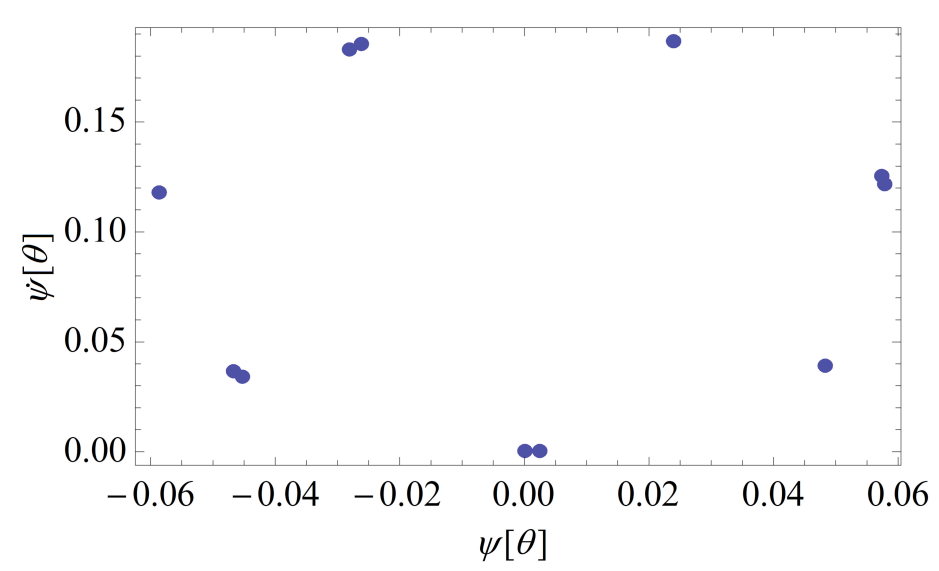

b)

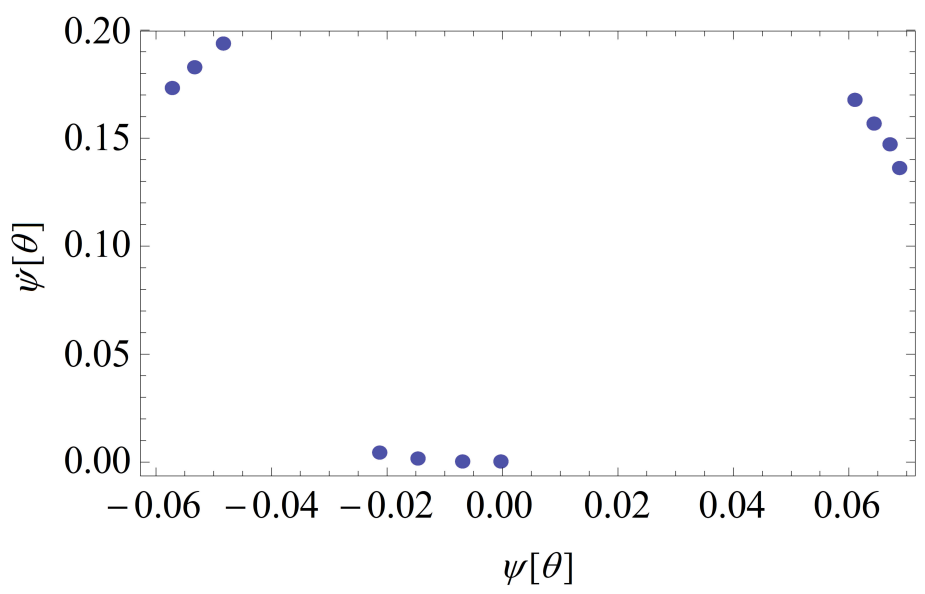

Figure 26: Poincaré map of the tether with initial conditions $\psi(0)=0 \mathrm{rad}, \dot{\psi}(0)=0 \mathrm{rad} / \mathrm{s}, \alpha(0)=0.1 \mathrm{rad}$ at $e=0.15$ for 230 orbits . (a) Rigid body tether and (b) flexible tether.

\section{Conclusions}

This study of a three dimensional model for a motorised momentum exchange tether has compared the response of the rigid body model with a flexible model. This comparative study between the three dimensional flexible model and the former rigid body models shows that the flexible model demonstrates a generally lower magnitude of response compared with that of the rigid body model. The application of torque increases the longitudinal displacement, but the transverse displacement shows a 
decaying phenomenon due to the stiffening effect of the rotating tether. This study also shows that a relationship between the planar and non-planar motions is found to be significant for the global motion of the tether, and dynamical analysis for two dimensional model has shown that the tether's flexibility has a significant effect on the its motion. The eccentricity and initial conditions are both found to influence the onset of chaos. However, non-zero initial conditions for the longitudinal and transverse displacements were not shown to have significant influence on the route to chaotic motion. Finally, in the analysis for three dimensional model, it also proved that the flexibility gives significant effect on the dynamics of the tether.

\section{References}

[1] Cartmell, M.P.: Generating Velocity Increments by Means of a Spinning Motorized Tether. 34th AIAA/ASME/SAE/ASEE Joint Propulsion Conference and Exhibit, Cleveland, AIAA Paper 98-3739, 20-24 June, Ohio, USA (1998)

[2] Bainum, P.M., and Kumar, V.K.: Optimal Control of the Shuttle-tethered System. Acta Astronautica, Vol. 7, 1333-1348 (1980) DOI: 10.1016/0094-5765(80)900107.

[3] Liaw, D.C., and Abed, E. H.: Stabilization of Tethered Satellites during Station Keeping. IEEE Transactions on Automatic Control, Vol. 35, 1186-1196 (1990) DOI: $10.1109 / 9.59804$.

[4] Netzer, E., and Kane, T.R.: Deployment and Retrieval Optimization of a Tethered Satellite System. Journal of Guidance Control and Dynamics, Vol. 6, 1085-1091 (1993) DOI: 10.2514/3.21131 
[5] Modi,V.J., Misra, A.K., and Geng, C.G.: Effect of Damping on the Control Dynamics of the Space Shuttle based tethered system. Proceedings of the Astrodynamic Conference, August 3-5, North Lake Tahoe, NV (1981).

[6] Puig-Suari, J., and Longuski J.M.: Modeling and Analysis of Orbiting Tethers in an Atmosphere. Acta Astronautica, Vol. 25, Issue 11, 679-686 (1991) DOI: 10.1016/0094-5765(91)90044-6.

[7] Ziegler, S. W., and Cartmell, M.P.: Using Motorized Tethers for Payload Orbital Transfer. Journal of Spacecraft and Rockets, Vol. 38, No.6, 904-913 (2001) DOI: $10.2514 / 2.3762$.

[8] Fujii, H., and Ishijima, S.: Mission Function Control for Deployment and Retrieval of a Subsatellite. Journal of Guidance, Control, and Dynamics. Vol. 12, No. 2, 243-247 (1989) DOI: 10.2514/3.20397.

[9] He, Y., Liang, B., and Xu, W.: Study on the Stability of Tethered Satellite System. Acta Astronautica, Vol.68, (11-12), 1964-1972 (2011) DOI: 10.1016/ j.actaastro.2010.11.015.

[10] Iñarrea, M., Lanchares, V., Pascual, A., and Salas J.: Attitude Stabilization of Electrodynamic Tethers in Elliptic Orbits by Time-delay Feedback Control. Acta Astronautica, Vol. 96, 280-295 (2014) DOI: 10.1016/j.actaastro.2013.12.011.

[11] Kojima, H., Sugimoto, Y., and Furukawa, Y.: Experimental Study on Dynamics and Control of Tethered Satellite Systems with Climber. Acta Astronautica Vol. 69 (1-2), 96-108 (2011) DOI: 10.1016/j.actaastro.2011.02.009 
[12] Woo, P., and Misra, A.: Mechanics of very Long Tethered Systems. Acta Astronautica Vol. 87, 153-162, (2013) DOI: 10.1016/j.actaastro.2013.02.008

[13] Cai, Z., Li, X., and Wu, Z.: Deployment and Retrieval of a Rotating Triangular Tethered Satellite Formation Near Libration Points. Acta Astronautica, Vol. 98, 37-49 (2014) DOI : 10.1016/j.actaastro.2014.01.015

[14] Razzaghi, P., and Nima, A.: Study of the Triple-mass Tethered Satellite System under aerodynamic drag and J2 perturbations. Advance in Space Research, (2015) DOI: 10.1016/j.asr.2015.07.046.

[15] Banerjee, A.K.: Dynamics of Tethered Payloads with deployment Rate Control. Journal of Guidance, Control and Dynamics, Vol. 13, 759-762 (1990) DOI: $10.2514 / 3.25398$.

[16] No, T. S., and Cochran, Jr. J. E.: Dynamics and Control of a Tethered Flight Vehicle. Journal of Guidance, Control, and Dynamics, Vol. 18, No.1, 66-72 (1995) DOI: $10.2514 / 3.56658$.

[17] Danilin, A.N., Grishanina T.V., Shklyarchuk, F.N., and Buzlaev, D.V.: Dynamics of a Space Vehicle with Elastic Deploying Tether. Computers and Structures, Vol. 72, No.1, 141-147. (1999) DOI: 10.1016/S0045-7949(99)00039-5.

[18] Avanzini, G., and Fedi, M.: Refined Dynamical Analysis of Multi-tethered Satellite Formations. Acta Astronautica, Vol. 84, 36-48, (2013) DOI: 10.1016/ j.actaastro. 2012.10.031.

[19] Kunugi, K., Kojima, H., and Trivailo, P.: Modeling of Tape Tether Vibration and Vibration Sensing Using Smart Film Sensors. Acta Astronautica, Vol.107, 97-111 (2015) DOI: 10.1016/j.actaastro.2014.11.024 
[20] Biswell, B.L., Puig-Suari, J., Longuski, J.M, and Tragesser, S.G.:ThreeDimensional Hinged-rod Model for Elastic Aerobraking Tethers. Journal of Guidance, Control, and Dynamics, Vol. 21, 286-295 (1998) DOI: 10.2514/2.4234

[21] Cartmell, M. P., and McKenzie D.J.: A review of Space Tether Research. Progress in Aerospace Sciences, Vol. 44, No.1, 1-21(2008) DOI: 10.1016/j.paerosci.2007.08.002.

[22] Netzer, E., and Kane, T.R.: Estimation and Control of Tethered Satellite Systems. Journal of Guidance, Control, and Dynamics, Vol.18, 851-858 (1995) DOI: $0.2514 / 3.21469$.

[23] Kumar, K.D.: Review of Dynamics and Control of Nanoelectrodynamic Tethered Satellite Systems. Journal of Spacecraft and Rockets, Vol. 43, 705-720 (2006) DOI: $10.2514 / 1.5479$.

[24] Kim,E. and Vadali, S.R.: Modelling Issues Related to Retrieval of Flexible Tethered Satellite Systems. Journal Guidance,Control and Dynamic, Vol. 18, No.5, 1169-1176 (1995) DOI: 10.2514/3.21521

[25] Modi,V.J. and Misra, A.K.: On the Deployment Dynamics of Tether Connected Two-body System. Acta Astronautica, Vol.6, 1183-1197 (1979) DOI: 10.1016/0094-5765(79)90064-X.

[26] Beletsky, V.V, and Levin, E.M.: Dynamics of the Orbital Cable System. Acta Astronautica, Vol. 12, Issue 5, 285-291 (1985) DOI: 10.1016/0094-5765 (85)90063-3. 
[27] Misra, A. K., and Modi, V. J.: A Survey on the Dynamics and Control of Tethered Satellite System. Advance In the Astronautical Science, Vol. 62, 667-719 (1986).

[28] French, D., and Mazzoleni, A.: Modeling Tether-ballast Asteroid Diversion Systems, Including Tether Mass and Elasticity. Acta Astronautica, Vol. 103, 282306 (2014). DOI: 10.1016/j.actaastro.2014.04.014.

[29] Lee,T., Leok, M., and Harris McClamroch, N.: High-fidelity Numerical Simulation of Complex Dynamics of Tethered Spacecraft. Acta Astronautica, Vol 99, 215-230 (2014) DOI: 10.1016/j.actaastro.2014.02.021.

[30] Ziegler, S.W.:The Rigid Body Dynamic of Tethers in Space. PhD. Thesis, Department of Mechanical Engineering, University of Glasgow, Glasgow, UK (2003)

[31] Zukovic, M., Kovacic, I., and Cartmell M.P.: On the Dynamics of a Parametrically Excited Planar Tether. Communications in Nonlinear Science and Numerical Simulation, Vol. $26(1-3), 250-264$ (2015) DOI: 10.1016/j.cnsns. 2015.02.014

[32] Chen, Y., and Cartmell, M.P.: Multi-objective optimization of the Motorised Momentum Exchange Tether for Payload Orbital Transfer. IEEE Congress on Evolutionary Computation, 25-28 September, Singapore (2007).

[33] Ismail, N.A., and Cartmell, M.P.: Modelling of a Flexible Elastic Tether for the Motorised Momentum Exchange Tether Concept. RANM2009, 24 - 27 August, Kuala Lumpur, Malaysia (2009). 
[34] Awrejcewicz, J. and Krysko,V.A. Chaos in Structural Mechanics, Springer, Berlin, 2008. DOI: 10.1007/978-3-540-77676-5.

[35] Luo, C.J., Han, R.P.S, Tyc, G., Modi, V. J., and Misra, A.K.: Analytical Vibration and Resonant Motion of a Stretched Spinning Nonlinear Tether. Journal Guidance, Control and Dynamics, Vol. 19, No.5, 1162-117 (1996) DOI: 10.2514/ 3.21759 .

[36] Gandara, C.C., and Cartmell, M.P.: De-Spin of a Motorised Momentum Exchange Tether. XXXVII International Summer School on Advanced Problems in Mechanics, St Petersburg, Russia (2009).

[37] Cartmell, M.P, and D’Arrigo, M.C.: Simultaneous Forced and Parametric Excitation of a Space Tether, XXXIII International Summer School on Advanced Problems in Mechanics, St Petersburg, Russia (2005).

[38] Karasopoulos, H., and Richardson, D.L.: Chaos in the Pitch Equation of Motion for the Gravity Gradient Satellite. AIAA/AAS Astrodynamics Conference, AIAA Paper 92-4369-CP, 53-65, Hilton Head, South Carolina, USA (1992).

[39] Fujii, H.A, and Ichiki, W.: Nonlinear Dynamics of the Tethered Subsatellite System in the Station Keeping Phase. Journal Guidance, Control and Dynamics, Vol. 20, No.2, pp. 403-406 (1997). DOI: 10.2514/2.4057.

\section{Appendix A. Nomenclature}

$$
\begin{array}{ll}
\alpha & \text { non-planar angle } \\
\beta(x) \quad \text { mode shape function for lateral vibration }
\end{array}
$$


angular displacement of motor torque axis about the tether's longitudinal axis

$\varepsilon_{x}$

$\theta$

$\mu$

$\xi(x)$

$\rho$

$\tau$

$\phi(x)$

$\psi$

$\dot{\psi}$

$\omega$

$A$

$a$

E

e

$g_{0}$

$I_{i}$

$L$

$M_{m}$

$M_{P}$

$q_{1}(t)$

$q_{3}(t)$

$q_{2}(t)$

$R$ strain due to axial extension

true anomaly

Earth's gravitational constant

mode shape function for transverse vibration

density

motor torque

mode shape function for axial vibration

angular displacement of tether within the orbital angle

angular velocity of tether

argument of perigee

cross sectional area

semimajor axis for ecliptic orbit

modulus elasticity

orbit eccentricity

gravity constant of $9.81 \mathrm{~m} / \mathrm{s} 2$

mass moment of inertia

tether sub-span

mass of central facility

mass of payload

modal coordinate for axial vibration

modal coordinate for lateral vibration

modal coordinate for transverse vibration

distance from the central facility to the centre of the Earth 


\begin{tabular}{|c|c|}
\hline$R_{p}$ & orbital radius at perigee \\
\hline$R_{Y, \alpha}$ & rotation matrix for non-planar movement \\
\hline$R_{Z, \psi+\theta}$ & rotation matrix for planar movement \\
\hline$r_{m}$ & radius of central facility \\
\hline$r_{p}$ & radius of payload \\
\hline$r_{\mathrm{T}}$ & radius of tether's cross section \\
\hline$T$ & string's tension \\
\hline$T_{0}$ & centripetal forces \\
\hline$T_{\text {rot }}$ & kinetic energy for rotational motion \\
\hline$T_{\text {trans }}$ & kinetic energy for translational motion \\
\hline$U_{E 1, E 2}$ & elastic potential energy \\
\hline$U_{p}$ & total potential energy \\
\hline$v(x, t)$ & transverse displacement \\
\hline$w(x, t)$ & lateral displacement \\
\hline$X, Y, Z$ & $\begin{array}{l}\text { coordinate frame, with the origin at the centre of the } \\
\text { Earth }\end{array}$ \\
\hline$X_{o}, Y_{o}, Z_{o}$ & coordinate frame, with the origin at the centre of facility \\
\hline$x_{t l}, y_{t l}, z_{t 1}$ & $\begin{array}{l}\text { Cartesian components for position of point } \mathrm{P} \text { at upper } \\
\text { sub-span }\end{array}$ \\
\hline$x_{m m}, y_{m m}, z_{m m}$ & Cartesian components for the central facility \\
\hline$x_{P 2}, y_{P 2}, z_{P 2}$ & Cartesian components for the lower end mass \\
\hline$x_{P 1}, y_{P 1}, z_{P 1}$ & Cartesian components for the upper end mass \\
\hline
\end{tabular}




\section{Appendix B. Summary of Derivation for Generalized Force.}

Based on Figure 27, a summary of the derivation performed by Ziegler [21] for equation (15) is provided here. It starts by applying the theory of virtual work defined as follows,

$$
\delta W=F_{X} \delta x+F_{Y} \delta y+F_{Z} \delta z
$$

and considering the work done by all the non-conservative forces through appropriate virtual displacements, equations (B.2) and (B.3) are shown to apply,

$$
\begin{aligned}
& \delta W_{\alpha}=Q_{\alpha} \delta \alpha \\
& \delta W_{\psi}=Q_{\psi} \delta \alpha
\end{aligned}
$$

The generalized forces with respect to the generalised coordinates $\alpha$ and $\psi$ are given by,

$$
\begin{gathered}
Q_{\alpha}=F_{x} \frac{\partial x}{\partial \alpha}+F_{y} \frac{\partial y}{\partial \alpha}+F_{z} \frac{\partial z}{\partial \alpha} \\
Q_{\psi}=F_{x} \frac{\partial x}{\partial \psi}+F_{y} \frac{\partial y}{\partial \psi}+F_{z} \frac{\partial z}{\partial \psi}
\end{gathered}
$$




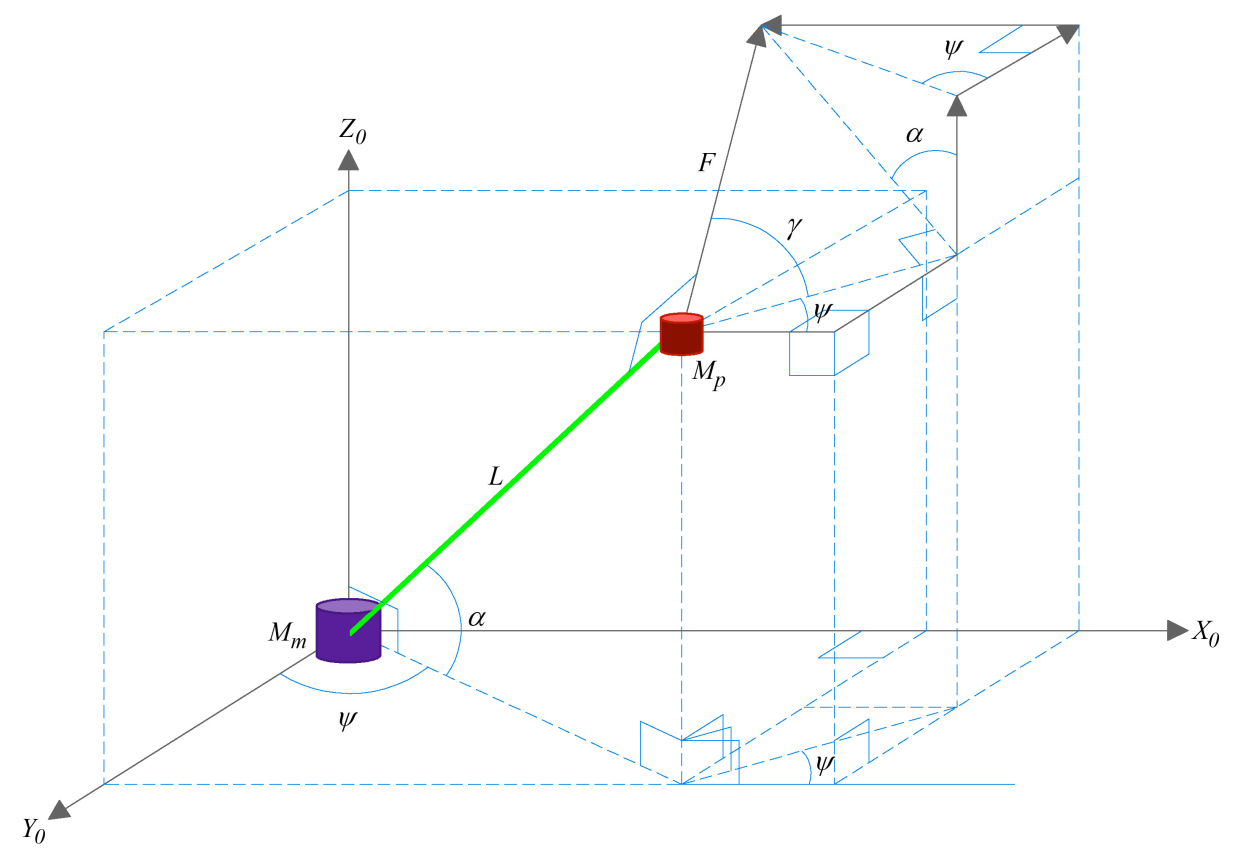

Figure 27 : Components of forces [21]

The components of force in the $\mathrm{x}, \mathrm{y}$ and $\mathrm{z}$ directions are,

$F_{x}=-F \cos \gamma \sin \psi-F \sin \gamma \sin \alpha \cos \psi$

$F_{y}=F \cos \gamma \cos \psi-F \sin \gamma \sin \alpha \cos \psi$

$F_{z}=F \sin \gamma \cos \alpha$

and so partially differentiating the Cartesian component of the end mass with respect to $\alpha$ and $\psi$ and substituting from equation (B.6), (B.7) and (B.8) into (B.4) and (B.5) gives the generalised forces as

$Q_{\psi}=\tau \cos \gamma \cos \alpha$

$Q_{\alpha}=\tau \sin \gamma$ 